\title{
Gold nanoparticles enlighten the future of cancer theranostics
}

\author{
This article was published in the following Dove Press journal: \\ International Journal of Nanomedicine \\ 22 August 2017 \\ Number of times this article has been viewed
}

\author{
Jianfeng Guo' \\ Kamil Rahme ${ }^{2-4}$ \\ Yan $\mathrm{He}$ \\ Lin-Lin $\mathrm{Li}^{5}$ \\ Justin D Holmes ${ }^{3,4}$ \\ Caitriona M O'Driscoll ${ }^{6}$ \\ 'School of Pharmaceutical Sciences, \\ Jilin University, Changchun, China; \\ ${ }^{2}$ Department of Sciences, Faculty of \\ Natural and Applied Science, Notre \\ Dame University (Louaize), Zouk \\ Mosbeh, Lebanon; ${ }^{3}$ Department \\ of Chemistry, Tyndall National \\ Institute, University College Cork, \\ Cork, ${ }^{4}$ AMBER@CRANN, Trinity \\ College Dublin, Dublin, Ireland; ${ }^{5}$ The \\ First Hospital of Jilin University, \\ Changchun, China; ${ }^{6}$ Pharmacodelivery \\ Group, School of Pharmacy, University \\ College Cork, Cork, Ireland
}

\begin{abstract}
Development of multifunctional nanomaterials, one of the most interesting and advanced research areas in the field of nanotechnology, is anticipated to revolutionize cancer diagnosis and treatment. Gold nanoparticles (AuNPs) are now being widely utilized in bioimaging and phototherapy due to their tunable and highly sensitive optical and electronic properties (the surface plasmon resonance). As a new concept, termed "theranostics," multifunctional AuNPs may contain diagnostic and therapeutic functions that can be integrated into one system, thereby simultaneously facilitating diagnosis and therapy and monitoring therapeutic responses. In this review, the important properties of AuNPs relevant to diagnostic and phototherapeutic applications such as structure, shape, optics, and surface chemistry are described. Barriers for translational development of theranostic AuNPs and recent advances in the application of AuNPs for cancer diagnosis, photothermal, and photodynamic therapy are discussed.
\end{abstract}

Keywords: multifunctional gold nanoparticles, cancer bioimaging, cancer photothermal and photodynamic therapy

\section{Introduction}

Cancer, one of the leading causes of mortality worldwide, has caused approximately 8.8 million deaths in 2015 (www.who.int). The number of people who are diagnosed with this malignancy is expected to rise to 22 million annually in the next 2 decades (www.who.int). Despite the increased knowledge about the causes of cancer and the improved interventions to prevent and manage the disease, survival rates are still low mainly due to the delay in diagnosis, lack of effective therapeutics, and high incidence of relapse.

As an emerging concept that facilitates simultaneous diagnosis and treatment, the implementation of theranostic nanomaterials (ie, metal and silica nanoparticles [NPs], liposomes, dendrimers, quantum dots, and carbon nanotubes) has great potential for improved cancer treatment and reduced side effects. ${ }^{1-6}$ Among these, gold NPs (AuNPs) exhibit favorable physical properties and tailored surface functionalization, providing a potential platform for developing cancer theranostics. This review provides a comprehensive overview of AuNPs as emerging nanomaterials for future cancer theranostics. In this regard, the key physicochemical properties of AuNPs such as structure, shape, optics, and surface chemistry are discussed. In addition, various AuNP-based diagnostic and phototherapeutic strategies under investigation are critically evaluated, with a particular emphasis on those developed to overcome delivery barriers. 
Key properties of AuNPs for diagnosis and phototherapy Types of AuNPs

Colloidal AuNPs were first produced in 1857 by Faraday, ${ }^{7}$ where the "fine particles" were formed from the reduction of gold chloride by phosphorus and the stabilization of AuNPs by carbon disulfide. In 1951, Turkevich et $\mathrm{al}^{8}$ reported the formation of colloidal AuNPs using trisodium citrate $\left(\mathrm{HOC}(\mathrm{COONa})\left(\mathrm{CH}_{2} \mathrm{COONa}\right)_{2}\right)$ to reduce tetrachloroauric acid (gold [III] chloride [ $\left.\mathrm{HAuCl}_{4}\right]$ ) in water, and later Frens ${ }^{9}$ improved the formation using a slightly modified method. Recently, AuNPs have been produced with various sizes and shapes (ie, gold nanospheres, nanorods, nanocages, nanoshells, and nanostars), which are dependent upon the synthetic methods adopted for their preparation (Figure 1; a summary of synthetic approaches to obtain various gold nanostructures has been described previously). ${ }^{10-14}$
A

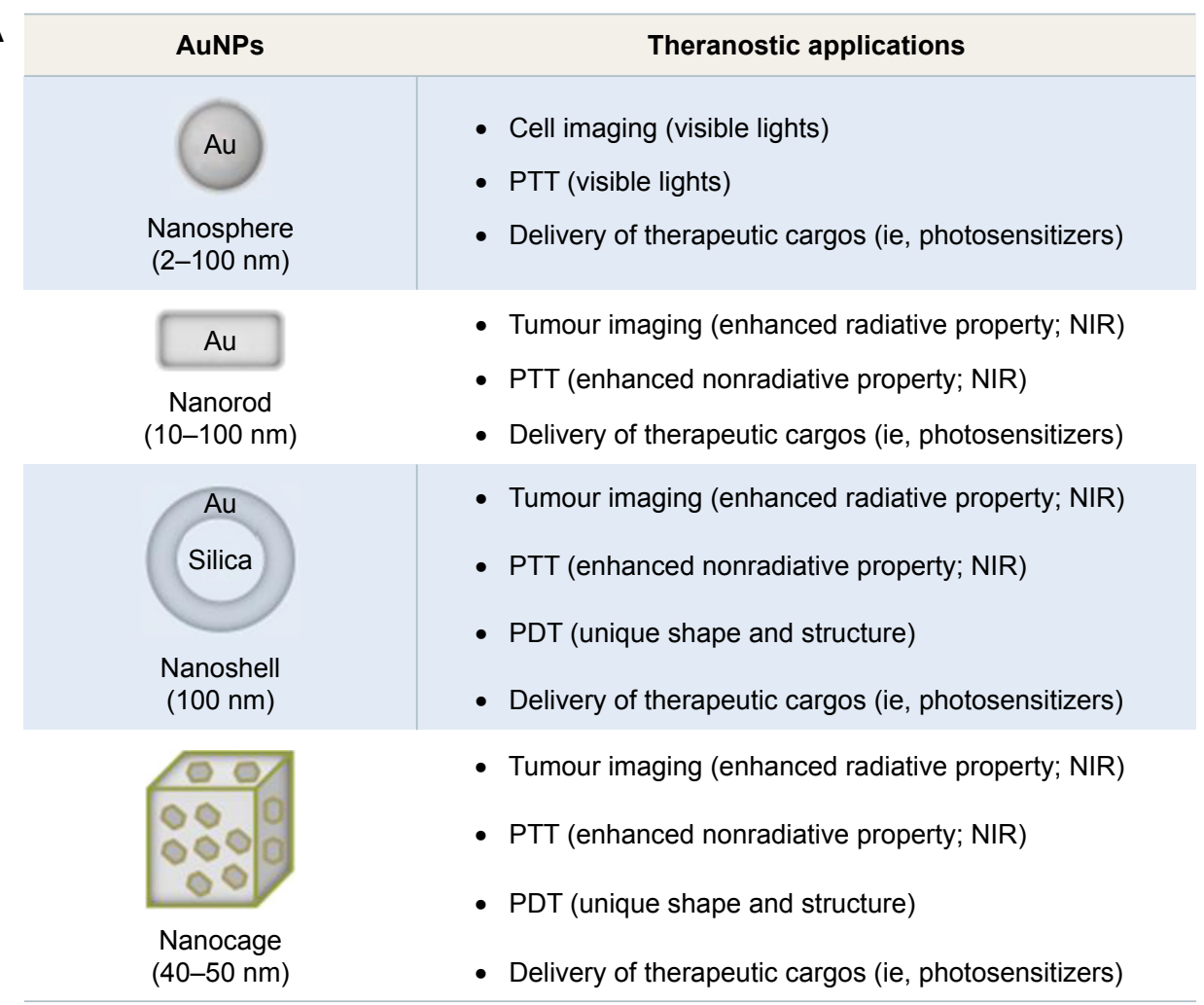

B

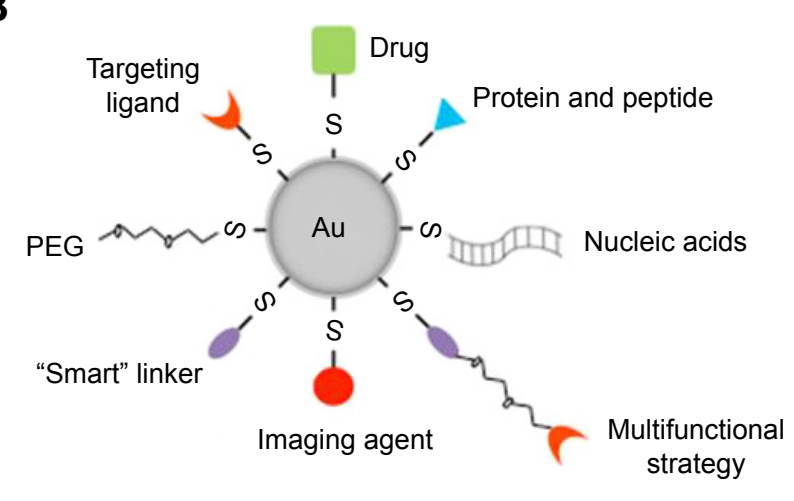

- Improved stability

- Enhanced EPR effect

- Cell-specific targeting

- Enhanced NIR activation

- Controlled drug release

Figure I Development of theranostic AuNPs in the treatment of cancer.

Notes: (A) Commonly used AuNPs can be categorized depending on the particle shape, including Au nanospheres, nanorods, nanoshells, and nanocages. These AuNPs with tunable optical and electronic properties and easy surface functionalizations have presented great potential for cancer bioimaging, PTT/PDT, and targeted drug delivery. (B) Functional components including stealth coating materials, bioresponsive moieties, bioactive targeting ligands, bioimaging agents, and therapeutic cargos can be integrated into one system, to achieve multifunctional AuNPs for future cancer treatment.

Abbreviations: AuNPs, gold nanoparticles; EPR, enhanced penetration and retention; NIR, near-infrared; PDT, photodynamic therapy; PEG, polyethylene glycol; PTT, photothermal therapy. 


\section{Gold nanospheres}

Gold nanospheres (particle size 2-100 nm) are generally prepared after reducing $\mathrm{HAuCl}_{4}$ with the assistance of different reducing agents under various temperature and pressure. Trisodium citrate (sometimes referred to simply as sodium citrate), for example, is a commonly used reducing and stabilizing agent, which is capable of generating monodisperse gold nanospheres with different particle sizes by adjusting the concentration of citrate. ${ }^{11}$

The seeding growth strategy has been used to improve monodispersity and avoid the formation of large AuNPs with irregular shapes (diameter $>50 \mathrm{~nm}$ ), whereby large spherical AuNPs are produced by reducing $\mathrm{HAuCl}_{4}$ or $\mathrm{Au}$ (III)-surfactant complexes onto the surface of preexisting seeds using reducing agents such as hydroxylamine hydrochloride,,${ }^{15,16}$ ascorbic acid,,${ }^{17}$ 2-mercaptosuccinic acid, ${ }^{18}$ hydroquinone, ${ }^{19}$ and hydrogen peroxide..$^{20}$

\section{Gold nanorods (GNRs)}

GNRs were first synthesized by Foss et al, ${ }^{21}$ Martin, ${ }^{22}$ and Perez-Juste et $\mathrm{a}^{23}$ using the template method. They are generally synthesized with a size ranging from $10 \mathrm{~nm}$ to $100 \mathrm{~nm}$ and an aspect ratio between 1 (sphere) and 7 with a corresponding longitudinal plasmon of $\sim 1,050 \mathrm{~nm}$. However, the yield is low due to the fact that only one monolayer of nanorods is produced using the template approach.

Alternatively, the seed growth method is also used to synthesize GNRs. ${ }^{23}$ In this method, gold seeds are prepared by reducing the gold salt with a strong reducing agent (ie, sodium borohydride). These seeds that provide the nucleation sites are subsequently added to the aqueous surfactant media containing the gold salt and the reducing agents (ie, ascorbic acid and hexadecyltrimethylammonium bromide [CTAB]) for the growth steps. ${ }^{24,25}$ Additional nucleation during the growth stage can be inhibited by controlling the growth conditions such as 1) the rate of addition of reducing agents to the gold seed and gold salt solution and 2) the reduction potential of reducing agents. As a result, the aspect ratios of nanorods (the ratio of the longer side to the shorter side) can be controlled by using different amounts of gold seeds relative to the precursor.

\section{Gold nanocages}

Gold nanocages are hollow nanostructures (particle size $\sim 40-50 \mathrm{~nm}$ ) which can be prepared with controllable pores on the surface via the galvanic replacement reaction between truncated silver $(\mathrm{Ag})$ nanocubes and $\mathrm{HAuCl}_{4}{ }^{26}$ Silver nanostructures with controlled shapes can be generated via the polyol reduction, where $\mathrm{AgNO}_{3}$ is reduced by ethylene glycol to generate Ag atoms followed by nanocrystals or seeds. Silver nanostructures are utilized as the sacrificial template and can be transformed into AuNPs with hollow structures via the galvanic replacement. ${ }^{26}$ The molar ratio of $\mathrm{Ag}$ to $\mathrm{HAuCl}_{4}$ can be regulated to manipulate the diameter and wall thickness of resultant gold nanocages. ${ }^{26}$

\section{Gold nanoshells}

Gold nanoshells are normally composed of dielectric core materials (ie, silica and polystyrene) coated by a thin gold layer. Core materials such as silica and polystyrene have been widely used to provide high stability and monodispersity. ${ }^{27}$ These core materials can be tailored by varying the dimensions of the core and/or the shell; normally, the core has a diameter $\sim 100 \mathrm{~nm}$ and a thin shell of gold about several nanometers $(\sim 1-20 \mathrm{~nm}){ }^{28,29}$

Modification of the core surface with a bifunctional ligand that enhances the shell coverage is a common method for the preparation of gold nanoshells. In the case of silica, the core surface is modified by 3-aminopropyltriethoxysilane (APS) with both ethoxy and amine groups. The ethoxy group binds covalently to silica surface through the hydroxyl group, while the amine group attaches to gold seeds. In addition, bifunctional linkers such as 3-aminopropyltrimethoxysilane (APTMS) and 3-mercaptopropyltrimethoxysilane (MPTMS) have also been used to modify the silica, leading to aminoand thiol-functionalized surfaces that can efficiently attach to gold seeds. As a result, the complete shell is formed by aging the gold attached onto the silica core. ${ }^{30}$

\section{Optical characteristics of AuNPs}

It is known that the oscillating electromagnetic field of light induces a collective coherent oscillation of the free electrons (also known as conduction band electrons) of the metal. ${ }^{31}$ The amplitude of the oscillation that reaches a maximum at a specific frequency is termed the surface plasmon resonance (SPR). ${ }^{31} \mathrm{~A}$ strong absorption of the incident light is induced by the SPR and can be measured using an ultraviolet (UV)visible absorption spectrometer. ${ }^{32}$ The SPR band of noble metals (ie, gold and silver) is known to be much stronger than other metals. ${ }^{32}$ The SPR wavelength of AuNPs can be tuned from the visible to the near-infrared (NIR) region by changing the size, shape, and structure of AuNPs, as theoretically described by the Mie theory. ${ }^{32}$

Two main processes, namely, absorption and scattering, occur when the light passes through matter resulting in the energy loss of electromagnetic wave..$^{33}$ The scattered light has the same frequency as the incident light (it is termed as Rayleigh scattering) or a shifted frequency (it is termed as 
Raman scattering). ${ }^{33}$ AuNPs can significantly enhance the light scattering, ie, five- to six-fold stronger than most strongly absorbing organic dyes and higher than the emission of most strongly fluoresceins, ${ }^{34}$ which makes AuNPs very promising imaging and detection platforms for cancer, as described in the "AuNPs for cancer diagnosis" section.

The scattering properties are highly dependent on the size and shape of AuNPs, which are given in the following sections.

\section{Particle size}

AuNPs with particle sizes of $\sim 40 \mathrm{~nm}$ may be easily detected down to a particle concentration of $10^{-14} \mathrm{M} \cdot{ }^{35,36}$ Moreover, the scattering of $\sim 60 \mathrm{~nm}$ AuNPs is $\sim 100$-fold stronger than the emission of a fluorescein. ${ }^{36}$ Likewise, $\sim 70 \mathrm{~nm}$ AuNPs can scatter orders of magnitude stronger than that of a polystyrene sphere of the same size..$^{37}$ It has been reported that $\sim 30-100 \mathrm{~nm}$ AuNPs can be detected under a microscope using dark-field illumination conditions (only the light scattered from indirect illumination of the sample is detected).$^{38}$ Recently, Jain et al ${ }^{34}$ and Lee and El-Sayed ${ }^{39}$ have substantially studied the relationship between the optical absorption/scattering and the size of AuNPs using the Mie theory. They reported that the total extinction of $\sim 20 \mathrm{~nm}$ AuNPs was mostly contributed by absorption; when the particle size of AuNPs was $\sim 40 \mathrm{~nm}$, they initiated scattering, and when the particle size was $\sim 80 \mathrm{~nm}$, the extinction of AuNPs was contributed by absorption and scattering in a very similar degree. ${ }^{34,39}$ This fact that the ratio of scattering to absorption increases significantly for larger size of particles can guide the development of AuNPs for bioimaging.

\section{Particle shape}

The optical properties of AuNPs can also be tuned by shape ${ }^{40-42}$ When the shape of AuNPs is changed from sphere to rod, the SPR will be split into two bands, namely, a strong band in the NIR region and a weak band in the visible region, as predicted by the Gans theory. The strong band, also referred to as the longitudinal band, results from electron oscillations along the long axis. The weak band, also known as the transverse band, is similar to the wavelength of gold nanospheres. Tong et $\mathrm{al}^{43}$ reported that the longitudinal band of GNRs was red shifted largely by increasing the aspect ratios (length/width), which causes the color change from blue to red. In addition, Lee and El-Sayed ${ }^{44}$ have shown that when the aspect ratio of GNRs was increased, light scattering was significantly enhanced.
It is known that the SPR of gold nanocages may be tuned to the NIR region with specified wavelengths..$^{45}$ For instance, the particle size of gold nanocages is generally $\sim 50 \mathrm{~nm}$ edge width with several nanometer walls and holes for an SPR wavelength of $\sim 800 \mathrm{~nm} .{ }^{46}$

\section{Particle composition}

It is known that the composition of NPs (particularly semiconductor NPs such as quantum dots) is also able to affect the scattering properties ${ }^{47}$ recently, several studies have focused on the scattering properties dependent on the composition of Au nanocomplexes. ${ }^{48-50}$ Jain et a $l^{34}$ have investigated the scattering properties of the core-shell composition in silica-Au nanoshells using Mie theory and discrete dipole approximation method. Results show that the thicknesses of the core and the shell and the radius ratio of core/shell significantly influenced the optical characteristics such as the resonance wavelength, the extinction cross-section, and the ratio of scattering to absorption. ${ }^{34}$ In addition, it was reported that the SPR wavelength of silica-Au nanoshells may be changed by controlling the $\mathrm{Au}$ shell thickness; for example, when the shell thickness was decreased from 20 to $5 \mathrm{~nm}$, the SPR was red shifted $\sim 300 \mathrm{~nm}$, which most likely results from the increased coupling between the inner and outer shell surface plasmons for thinner shell particles. ${ }^{30}$

\section{Surface functionalization of AuNPs}

The surface chemistry of gold makes AuNPs a promising platform for biomedical applications (Figure 1). ${ }^{11}$ Brust et $\mathrm{al}^{51}$ exploited the high affinity of thiol for gold to produce AuNP-thiolates (Au-S); this was achieved using $\mathrm{HAuCl}_{4}$, thiol, tetraoctylammonium bromide, and $\mathrm{NaBH}_{4}$ in watertoluene and was stabilized via Au-S-stabilizer bonds. These NPs were dispersed in organic solvent and required further phase transfer or ligand exchange to transfer them into water. An essential purification step to remove impurities for use in biological application was also necessary.

In addition, ligands containing amine and phosphine groups, which also have high affinity with the surface of gold, have been used as efficient stabilizing agents. ${ }^{52}$ For example, cationic surfactant-free AuNPs ( $\sim 2-200 \mathrm{~nm})$ have been developed that were synthesized in water using a seed growth method, in the presence of L-cysteine methyl ester hydrochloride $\left(\mathrm{HSCH}_{2} \mathrm{CH}\left(\mathrm{NH}_{2}\right) \mathrm{COOCH}_{3} \cdot \mathrm{HCl}\right)$ as a capping agent. ${ }^{53}$ Furthermore, Chhour et $\mathrm{al}^{54}$ have functionalized AuNPs using a group of thiol and amine ligands, including 11-mercaptoundecanoic acid (11-MUDA), 16-mercaptohexadecanoic acid (16-MHDA), polyethylenimine (PEI), 
4-mercapto-1-butanol (4-MB), and 11-mercaptoundecyltetra (ethylene glycol) (MTEG). These ligands enhanced the AuNP stability and provided different surface functionalities which can influence cell uptake and cytotoxicity. ${ }^{54}$ Among them, the 11-MUDA AuNPs were used to monitor the recruitment of monocytes into atherosclerotic plaques in a disease mouse model using X-ray computed tomography (CT), potentially allowing detection of monocyte recruitment in the presence of emerging atherosclerosis therapies. ${ }^{54}$ In addition, Liu et $\mathrm{al}^{55}$ have recently stabilized AuNPs using thiol-polyethylene glycol (SH-PEG) at two different ratios of thiol to PEG (1:1 and 1:2), significantly improving the AuNP stability in biological media. When AuNPs with higher PEG content were intravenously injected, they were found to accumulate in the liver at a lower level relative to counterparts with lower PEG content. ${ }^{55}$ These results suggest that when designing AuNPs, a rational ratio between the anchoring group (ie, thiol) and the hydrophilic group (ie, PEG) should be carefully considered, this is important for integrating the properties of NPs in certain bio-related application.

Targeting ligands ${ }^{56-60}$ may be modified onto the AuNP surface to specifically deliver therapeutic cargos into tissues and organs. Recently, tumor-targeted mesoporous silicaencapsulated GNRs (GNRs@ $\mathrm{mSiO}_{2}$ ) for chemotherapy and photothermal therapy (PTT) have been developed by Shen et al. ${ }^{61}$ In this study, RGD peptides, a targeting ligand for $\alpha_{\mathrm{v}} \beta_{3}$ integrin receptors that are known to overexpress on several cancer cells, were conjugated to the terminal groups of PEG on GNRs@mSiO 2 (namely,pGNRs@mSiO $2-\mathrm{RGD}$ ). The pGNRs@ $\mathrm{mSiO}_{2}$-RGD presented significant stability in bioenvironments and efficient loading of doxorubicin (DOX, an antitumor chemotherapeutics). Following the NIR irradiation, the combination of photothermal ablation and DOX-mediated cytotoxicity using pGNRs@ $\mathrm{mSiO}_{2}$-RGD resulted in significant tumor reduction in subcutaneous xenografted mice. ${ }^{61}$ In addition, it has been reported that PEI-capped AuNPs (Au-PEI) were conjugated with anisamide (AA, which is known to bind to the sigma receptor overexpressed on prostate cancer cells) ${ }^{62,63}$ to produce the AA-targeted AuNPs (Au-PEI-AA). ${ }^{59}$ As a result, Au-PEI-AA facilitated siRNA uptake into prostate cancer PC-3 cells via binding to the sigma receptor and achieved efficient downregulation of the targeted oncogene. ${ }^{59}$

In addition to the aforementioned stabilizing and targeting ligands, the gold surface can also be modified using several suitable imaging agents, photosensitive molecules, and bioactive/bioresponsive moieties (ie, $\mathrm{pH}$-sensitive linkers, matrix metalloproteinase [MMP]-sensitive linkers, temperature-sensitive linkers, fusogenic/synthetic peptides, and endosomal membrane-disruptive materials) to achieve multifunctional AuNPs for cancer diagnosis and phototherapy, which are discussed in the following sections.

\section{AuNPs for cancer diagnosis Spectroscopic cancer imaging}

For wavelengths $>650$ and $<2,000 \mathrm{~nm}$, the tissue absorption is weak, so the NIR light (wavelength from 700 to $2,500 \mathrm{~nm}$ ) is normally chosen to image tumor deeply within the body. It is worth noting that the penetration depth of NIR light into tissues is highly dependent on the tissue type, the wavelength, and the condition of the incident beam (ie, the laser power, irradiation time, and time interval). ${ }^{64-66}$ For example, it was shown that no penetration was found in the skin, skull, or brain for NIR light with low-power laser; however, $0.45 \%-2.90 \%$ of $810 \mathrm{~nm}$ NIR light at high power (10-15 W) was delivered into $3 \mathrm{~cm}$ of the aforementioned tissues. ${ }^{66}$

AuNPs on their own may act as an NIR-active imaging probe for cancer detection facilitating whole-body scans due to the unique optical properties. The use of targeted AuNPs as the contrast agent was demonstrated by Sokolov et al, ${ }^{37}$ where AuNPs were conjugated with an antibody against the epidermal growth factor receptor (EGFR, it is known to overexpress on many cancers). These AuNP conjugates were used for detecting cancer cells using a scanning confocal microscope in the reflectance mode with a $647 \mathrm{~nm}$ laser to excite the SPR of AuNPs; as a result, cells with AuNP conjugates were clearly imaged on a dark background. ${ }^{37}$ El-Sayed et $\mathrm{al}^{67}$ improved the use of AuNPs $(\sim 35 \mathrm{~nm})$ as the contrast agent via dark-field microscopy. Following excitation by white light, only the wavelength of light corresponding to the maximum of the SPR of AuNPs was displayed intensely, where a bright image of cells with AuNPs could be seen on a dark background. ${ }^{67}$ Moreover, cetuximab (CET, a chimeric monoclonal antibody against the EGFR)-conjugated PEGylated GNRs (CET-pGNRs) was developed for cancer imaging in xenografted mice. ${ }^{68}$ The results of in vivo NIR absorption imaging show that specific targeting of CET-pGNRs to the tumor region was evident by a significant increase in the absorption signal. The biodistribution data also show that the amount of AuNPs in tumor tissues from mice injected with CET-pGNRs was eightfold greater than that recorded by nontargeted pGNRs, confirming the results from the NIR absorption imaging. These indicate that CET-pGNRs can specifically target tumor 
tissues with high specificity and provide a potential tool for NIR-based cancer diagnosis.

Recently, the photoacoustic imaging has taken advantage of plasmonic systems, such as AuNPs with various sizes and shapes. ${ }^{69}$ Plasmon resonances of AuNPs can be tuned to enhance the optical response, ${ }^{70,71}$ which can give rise to heat conversion with high efficiency and to the subsequent pressure wave generating the photoacoustic signal. Indeed, these properties have been utilized to develop AuNPs as contrast agents for the photoacoustic imaging. ${ }^{72}$ Recently, an amphiphilic GNR coated with PEG and poly(lactic-coglycolic acid) (PLGA; AuNR.PEG.PLGA) was developed for the photoacoustic imaging in xenografted mice. ${ }^{73}$ The AuNR.PEG.PLGA could self-assemble into vesicles with the AuNRs embedded in the shell formed by the PLGA and PEG extending into the aqueous environments to stabilize the structure. Furthermore, the in vivo two-dimensional (2D) and three-dimensional (3D) photoacoustic images show that the strong plasmonic coupling of GNRs in the vesicles induced a high photothermal effect and a photoacoustic signal, which may potentially be used for image-guided phototherapy in the future. ${ }^{73}$

In addition, AuNPs have also been utilized as highquality CT imaging agents due to better X-ray attenuation properties (atomic number $Z=79$; $\mathrm{k}$-edge value $=80.7 \mathrm{keV}$ ) than that of iodinated CT contrast agents. ${ }^{74,75}$ In addition, the conventional small-molecular CT contrast agents are rapidly cleared by the kidney resulting in short imaging times, whereas the gold surface can be modified with biological stabilizing groups (ie, PEG) to improve the pharmacokinetic properties ${ }^{76}$ which is beneficial for cancer imaging. Recently, a folic acid (FA)-targeted gold nanosphere (FA-PEG-PEI-AuNPs) was developed using PEI and PEG as stabilizing ligands. ${ }^{77}$ The intravenous injection of FA-PEG-PEI-AuNPs into an overexpressed folate receptor tumor model resulted in significantly higher $\mathrm{CT}$ values in the tumor region compared with nontargeted PEG-PEI-AuNPs. In addition to the "enhanced penetration and retention" (EPR)-based passive tumor targeting, the FA-mediated active targeting was also able to significantly enhance the AuNP accumulation in tumor tissues, resulting in enhanced cancer CT imaging.

In addition, AuNPs are also known to enhance the Raman scattering signal of adjacent molecules, and therefore, surface-enhanced Raman spectroscopy (SERS) imaging aided by gold nanomaterials (spheres, rods, cubes, etc.) has also widely been used in the detection of viruses and cancer cells..$^{78,79}$

\section{Functionalized imaging agents for cancer detection}

Hybrid dual imaging technologies, including positron emission tomography (PET)/CT, PET/magnetic resonance imaging (MRI), and ultrasound/CT, have recently become available. ${ }^{80}$ Cancer diagnosis clearly benefits from these techniques due to multimodality, as a single agent may avoid the administration of multiple doses. However, the choice of imaging modality must be carefully considered since each one has its own advantages and limitations (ie, modalities with high sensitivity may have poor resolution).

AuNPs can be easily functionalized with additional imaging agents, and improvement in AuNP-based imaging systems may allow the observation of tissues not only on its basic anatomic configuration but also on the molecular level. ${ }^{42,81,82}$ Moreover, the real-time noninvasive monitoring potentially enables a rapid decision on whether the treatment regimen is effective in a given patient. ${ }^{40,83}$

Recently, Zhao et al ${ }^{84}$ have synthesized gold nanospheres doped with ${ }^{199} \mathrm{Au}$ atoms using a one-step procedure for single-photon emission CT (SPECT)/CT imaging in an orthotopic mouse xenograft of triple-negative breast cancer (TNBC). The high-stable radiolabeling ability resulted from the incorporation of ${ }^{199} \mathrm{Au}$ atoms into the crystal lattice of AuNPs. In addition, the ${ }^{199} \mathrm{Au}$-doped AuNPs were further modified with 1) PEGylation for favorable pharmacokinetics and 2) D-Ala1-peptide T-amide (DAPTA) for targeting $\mathrm{C}-\mathrm{C}$ chemokine receptor 5 (CCR5, a prognostic biomarker for breast cancer progression) ${ }^{84}$ Results demonstrate the suitability of ${ }^{199} \mathrm{Au}$ for SPECT/CT imaging and the potential of ${ }^{199} \mathrm{Au}$-AuNP-PEG-DAPTA for accurately detecting CCR5 in vivo.

Moreover, He et a ${ }^{85}$ have recently synthesized novel AuNPs for magnetic and CT dual-mode imaging in a mouse xenograft of colorectal cancer. $\mathrm{Fe}_{2} \mathrm{O}_{3}$ was first coated with $\mathrm{Au}$ nanoshell $\left(\mathrm{Fe}_{2} \mathrm{O}_{3} / \mathrm{AuNPs}\right)$, and subsequently the surface of the $\mathrm{Fe}_{2} \mathrm{O}_{3} /$ AuNPs was modified with lectins (sugar-binding proteins specifically bind to the carbohydrate moieties of the glycans on colorectal cancer cells) through bifunctional PEG-N-hydroxysuccinimide ester disulfide linkers (lectinPEG- $\mathrm{Fe}_{2} \mathrm{O}_{3} /$ AuNPs). The lectin-PEG- $\mathrm{Fe}_{2} \mathrm{O}_{3} /$ AuNPs demonstrated long circulation time, site-specific tumor distribution, and high-quality MRI and CT contrast enhancement effects in tumor tissues, suggesting that the resultant AuNPs are a promising contrast agent for dual-mode MRI/CT colorectal cancer imaging.

Furthermore, selected examples of AuNP-based systemic cancer imaging are provided in Table 1, including the types 
Table I A summary of studies on the in vivo use of gold nanocomplexes in systemic cancer imaging

\begin{tabular}{|c|c|c|c|c|c|}
\hline Functional ligand & Cancer type & In vivo model & Imaging technique & Comment & Reference \\
\hline \multicolumn{6}{|l|}{ Gold nanospheres } \\
\hline $\begin{array}{l}\text { Stabilizing ligand: PEG } \\
\text { and PEI } \\
\text { Targeting ligand: FA }\end{array}$ & Papilloma (KB cells) & $\begin{array}{l}\text { S.C. xenograft } \\
\text { mouse }\end{array}$ & CT & $\begin{array}{l}\text { The AuNP-PEI was modified with FA-linked } \\
\text { PEG, forming FA-targeted PEGylated AuNPs. } \\
\text { The resultant targeted AuNPs presented } \\
\text { potential role as a nanoprobe for CT imaging of } \\
\text { FA receptor-overexpressing xenografted tumor }\end{array}$ & 77 \\
\hline $\begin{array}{l}\text { Stabilizing ligand: PEG } \\
\text { fluorescent dye }\end{array}$ & $\begin{array}{l}\text { Colon carcinoma } \\
\text { (СT26 cells) }\end{array}$ & $\begin{array}{l}\text { S.C. allograft } \\
\text { mouse }\end{array}$ & CT & $\begin{array}{l}\text { The signal intensity and nanoprobe } \\
\text { accumulation of Au-NPAPF-PEG in the } \\
\text { tumor were up to } 24 \text { h post i.v. injection, } \\
\text { suggesting the role as a promising nanoprobe } \\
\text { for in vivo tumor-targeted CT imaging }\end{array}$ & 87 \\
\hline $\begin{array}{l}\text { Stabilizing ligands: PEG } \\
\text { and glucose } \\
\text { Targeting ligand: glucose }\end{array}$ & $\begin{array}{l}\text { Melanoma } \\
\text { (SKMEL23 cells) }\end{array}$ & $\begin{array}{l}\text { S.C. xenograft } \\
\text { mouse }\end{array}$ & CT & $\begin{array}{l}\text { The AuNP-labeled T cells were injected } \\
\text { intravenously to mice-bearing human melanoma } \\
\text { xenografts, and whole-body CT imaging allowed } \\
\text { examination of the distribution, migration, and } \\
\text { kinetics of T cells }\end{array}$ & 88 \\
\hline Stabilizing ligand: PEG & $\begin{array}{l}\text { Lung cancer (SPC- } \\
\text { Al cells) }\end{array}$ & $\begin{array}{l}\text { S.C. xenograft } \\
\text { mouse }\end{array}$ & CT & $\begin{array}{l}\text { Results suggest that PEGylated AuNPs can } \\
\text { be used as a promising contrast agent with } \\
\text { enhanced biocompatibility for CT imaging in } \\
\text { cancer diagnosis }\end{array}$ & 89 \\
\hline $\begin{array}{l}\text { Hybrid formulation: } \\
\text { mesoporous silica NPs } \\
\text { Emitter: }{ }^{64} \mathrm{Cu}\end{array}$ & Lung cancer & $\begin{array}{l}\text { Urethane- } \\
\text { induced lung } \\
\text { cancer mouse }\end{array}$ & PET & $\begin{array}{l}{ }^{64} \mathrm{Cu} \text {-labeled gold/mesoporous silica hybrid } \\
\mathrm{NPs} \text { can successfully detect the existence of } \\
\text { clinically relevant spontaneous lung tumors in } \\
\text { a urethane-induced lung cancer mouse model } \\
\text { through PET imaging }\end{array}$ & 90 \\
\hline $\begin{array}{l}\text { Stabilizing ligand: PEG } \\
\text { Targeting ligand: TAT } \\
\text { Emitter: } \mathrm{Gd}^{3+}\end{array}$ & $\begin{array}{l}\text { Glioblastoma (U87 } \\
\text { cells) }\end{array}$ & $\begin{array}{l}\text { Orthotopic } \\
\text { xenograft } \\
\text { mouse }\end{array}$ & MRI & $\begin{array}{l}\text { Compared with the } \mathrm{Gd}^{3+} \text { chelate, TAT-Au } \\
\text { NP-Gd conjugates showed a } 2.2 \text {-fold higher } \\
\text { relaxivity and } 82 \text {-fold enhancement in } \\
\mathrm{Gd}^{3+} \text { cellular uptake, which allowed for sensitive } \\
\text { detection of the cancer cells via MRI }\end{array}$ & 91 \\
\hline $\begin{array}{l}\text { Stabilizing ligand: PEG } \\
\text { Targeting ligand: RGD } \\
\text { Emitter: }{ }^{125}\end{array}$ & $\begin{array}{l}\text { Glioblastoma (U87 } \\
\text { MG cells) }\end{array}$ & $\begin{array}{l}\text { S.C. xenograft } \\
\text { mouse }\end{array}$ & SPECT/CT & $\begin{array}{l}\text { In vivo SPECT/CT imaging results showed that } \\
\text { the }{ }^{125} \text {-labeled RGD-PEG-AuNP probes can } \\
\text { target the tumor site as soon as } 10 \text { min after } \\
\text { injection }\end{array}$ & 92 \\
\hline $\begin{array}{l}\text { Stabilizing ligand: PEG } \\
\text { Targeting ligand: } \\
\text { DAPTA }\end{array}$ & TNBC (4TI cells) & $\begin{array}{l}\text { Orthotopic } \\
\text { allograft mouse }\end{array}$ & SPECT/CT & $\begin{array}{l}\text { The synthesis of AuNPs was doped with }{ }^{199} \mathrm{Au} \\
\text { atoms into the crystal lattice of each AuNP, } \\
\text { which ensured the highest possible stability for } \\
\text { the radiolabel. When conjugated with DAPTA } \\
\text { for the CCR } 5 \text { receptor, the targeted AuNPs } \\
\text { resulted in the in vivo sensitive and specific } \\
\text { detection }\end{array}$ & 84 \\
\hline $\begin{array}{l}\text { Stabilizing ligand: GC } \\
\text { MMP sensitive linker: } \\
\text { MMP peptide } \\
\text { NIR dye: Cy5.5 }\end{array}$ & $\begin{array}{l}\text { Colorectal cancer } \\
\text { (HT-29 cells) }\end{array}$ & $\begin{array}{l}\text { S.C. xenograft } \\
\text { mouse }\end{array}$ & $\begin{array}{l}\mathrm{CT} \\
\text { NIR fluorescence } \\
\text { imaging }\end{array}$ & $\begin{array}{l}\text { The quenched Cy } 5.5 \text { was recovered by cleavage } \\
\text { of the peptide substrates upon exposure to the } \\
\text { active MMPs, which is overexpressed in tumor } \\
\text { tissue. As a result, the AuNPs simultaneously } \\
\text { provided CT images with high spatial resolution } \\
\text { and optical images with high sensitivity }\end{array}$ & 93 \\
\hline $\begin{array}{l}\text { Stabilizing ligand: PEG } \\
\text { Targeting ligand: FA } \\
\text { Emitter: } \mathrm{Gd}^{3+}\end{array}$ & Papilloma (KB cells) & $\begin{array}{l}\text { S.C. xenograft } \\
\text { mouse }\end{array}$ & $\begin{array}{l}\text { CT } \\
\text { MRI }\end{array}$ & $\begin{array}{l}\text { With the modification of PEG and the FA- } \\
\text { targeting ligand, the multifunctional AuNPs } \\
\text { were able to be used for dual-mode CT/MRI } \\
\text { of xenograft tumor models overexpressing FA } \\
\text { receptors }\end{array}$ & 94 \\
\hline $\begin{array}{l}\text { Photostability enhancer: } \\
\text { PB }\end{array}$ & $\begin{array}{l}\text { Colon } \\
\text { adenocarcinoma } \\
\text { (HT-29 cells) }\end{array}$ & $\begin{array}{l}\text { S.C. xenograft } \\
\text { mouse }\end{array}$ & $\begin{array}{l}\text { PAI } \\
\text { CT }\end{array}$ & $\begin{array}{l}\text { The AuNPs were coated with PB to form the } \\
\text { core/shell Au@PB NPs, which were found to be } \\
\text { an excellent photoabsorbing agent for both PTT } \\
\text { and PAI. The gold core ensured a remarkable } \\
\text { contrast enhancement for CT imaging }\end{array}$ & 95 \\
\hline
\end{tabular}


Table I (Continued)

\begin{tabular}{|c|c|c|c|c|c|}
\hline Functional ligand & Cancer type & In vivo model & Imaging technique & Comment & Reference \\
\hline $\begin{array}{l}\text { Stabilizing ligand: PEG } \\
\text { NIR dye: Cy5.5 }\end{array}$ & $\begin{array}{l}\text { Squamous } \\
\text { carcinoma } \\
\text { (SCC7 cells) }\end{array}$ & $\begin{array}{l}\text { S.C. allograft } \\
\text { mouse }\end{array}$ & $\begin{array}{l}\text { PAI } \\
\text { NIR fluorescence } \\
\text { imaging }\end{array}$ & $\begin{array}{l}\text { The resultant AuNPs showed high fluorescence } \\
\text { and } \mathrm{PAl} \text { signals in the tumor over time, which } \\
\text { peaked at the } 6 \mathrm{~h} \text { time point (tumor-to-normal } \\
\text { tissue ratio of } 3.64 \pm 0.5 \mathrm{I} \text { for optical imaging and } \\
2.5 \pm 0.27 \text { for } \mathrm{PAI} \text { ) }\end{array}$ & 96 \\
\hline
\end{tabular}

\section{GNR}

Stabilizing ligand: PEG

Targeting ligand: biotin

Squamous

carcinoma

(SCC7 cells)

Amphiphilic ligands:

PEG and PLGA

Stabilizing ligand: PEG

Targeting ligand: CET

Epithelial

carcinoma

(A43I cells)

Stabilizing ligand: PEG

NIR dye and

photosensitizer: $\mathrm{AlPcS}_{4}$

Stabilizing ligand:

PNIPAAmMA

MRI contrast agents:

$\mathrm{Fe}_{3} \mathrm{O}_{4} \mathrm{NPs}$

Stabilizing ligand: PEG

SERS reporters

Stabilizing ligand:

liposome

\section{Gold nanoshells}

Stabilizing ligand:

PPAA shell

Targeting ligand: CET

Emitter: ${ }^{89} \mathrm{Zr}$

Stabilizing ligand: PEG

Emitter: ${ }^{64} \mathrm{Cu}$

Targeting ligand: lectin

MRI contrast agents:

$\mathrm{Fe}_{3} \mathrm{O}_{4} \mathrm{NPs}$

Targeting ligand:

antibody (anti-NGAL)

MRI contrast agents:

$\mathrm{Fe}_{3} \mathrm{O}_{4} \mathrm{NPS}$

Stabilizing ligand: PEG

Emitter: $\mathrm{Gd}^{3+}$

Glioblastoma
(U87 MG cells)

Squamous carcinoma

(SCC7 cells)

SKOV3 cells)

Liver cancer

Epithelial

cells)

cells)
S.C. xenograft

PAI

mouse

S.C. xenograft

mouse

NIR fluorescence imaging

Glioma (C6 cells)

S.C. xenograft

mouse

PET

PAI

Ovarian cancer S.C. xenograft PA

(MDA-435S, HEY, mouse SERS imaging

(HepG2, Huh-7)

Orthotopic

xenograft

mouse

PAI

NIR fluorescence

imaging

carcinoma (A43

Head and neck

squamous cell

carcinoma (SCC4

Colorectal cancer

(SW620 cells)

Pancreatic cancer

(AsPC-I cells)

S.C. xenograft PET

mouse

S.C. xenograft PET/CT

rat

S.C. xenograft $M R I$

mouse

S.C. xenograft

mouse

MRI

NIR fluorescence imaging

Melanoma (BI6-

S.C. xenograft

mouse
MRI

X-ray imaging

Optical imaging
Under the photothermal/photoacoustic imaging,

the in vivo pharmacodynamic effect of resultant

GNRs could be monitored by precisely

controlling the irradiation time and intensity of

the NIR light

Amphiphilic AuNRs were prepared by grafting $\quad 73$

with PEG and PLGA forming vesicles. Enhanced

PA signals were due to the strong plasmonic

coupling of the gold in the vesicular shell

The NIR absorption images showed that the

68

relative total photon counts from targeted

Au nanorods in tumor tissue at $6 \mathrm{~h}$ were

I0-fold higher than those from nontargeted

counterparts

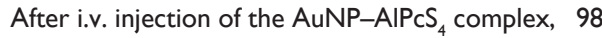

tumor sites were clearly identified on NIR

fluorescence imaging as early as I h after

injection

GNRs were coated with PNIPAAmMA

99

and $\mathrm{Fe}_{3} \mathrm{O}_{4}$ NPs using a simple LbL method,

demonstrating the accurate tumor location

using dual MRI and PAI

PEGylated Au nanorods allowed presurgical PAI $\quad 100$

visualization of a tumor for locoregional staging

as well as intraoperative SERS imaging for

complete resection of tumor margins

ICG-loaded liposome-Au nanorods exhibit

favorable biocompatibility, high stability, and

enhanced dual-model imaging signal

PET studies showed that the resultant

AuNPs-PPAA-CET- ${ }^{89} \mathrm{Zr}$ provided high tumor-

to-background ratio, suggesting a valuable tool

for theranostic purposes

The in vivo distribution of ${ }^{64} \mathrm{Cu}-\mathrm{Au}$ nanoshells

was monitored using PET/CT imaging at various

time points after i.v. injection

The lectin- $-\mathrm{Fe}_{2} \mathrm{O}_{3} @ \mathrm{Au}$ nanoshells showed great

potential for dual-mode MRI and CT imaging of colorectal cancer in vivo

Antibody-conjugated Au nanoshells

specifically targeted pancreatic cancer

cells in vivo providing contrast for both NIR

fluorescence and T2-weighted MRI with high

tumor contrast

The $\mathrm{Gd}^{3+}$-conjugated Au-silica nanoshells

105

showed great potential for multimode MRI,

$X$-ray imaging, and optical imaging of melanoma in vivo 
Table I (Continued)

\begin{tabular}{|c|c|c|c|c|c|}
\hline Functional ligand & Cancer type & In vivo model & Imaging technique & Comment & Referenc \\
\hline $\begin{array}{l}\text { MMP-triggering ligand: } \\
\text { gelatin } \\
\text { MRI contrast agent: } \\
\mathrm{Fe}_{3} \mathrm{O}_{4}\end{array}$ & $\begin{array}{l}\text { Hepatoma }(\mathrm{H} 22 \\
\text { cells) }\end{array}$ & $\begin{array}{l}\text { S.C. allograft } \\
\text { mouse }\end{array}$ & $\begin{array}{l}\mathrm{CT} \text { and PAT imaging } \\
\text { and } \mathrm{MRI}\end{array}$ & $\begin{array}{l}\text { A bio-eliminable MPNA, assembled from } \mathrm{Fe}_{3} \mathrm{O}_{4} \\
\text { nanocluster and gold nanoshell, could respond } \\
\text { to the local microenvironment with acidic pH } \\
\text { and enzymes in tumors, collapse into small } \\
\text { molecules and discrete NPs, and finally be } \\
\text { cleared from the body }\end{array}$ & 106 \\
\hline \multicolumn{6}{|l|}{ Gold nanoclusters } \\
\hline $\begin{array}{l}\text { Stabilizing ligand: BSA } \\
\text { Targeting ligand: } \\
\text { methionine } \\
\text { NIR dye: hydrophilic } \\
\text { ICG }\end{array}$ & $\begin{array}{l}\text { Breast cancer } \\
\text { (MDA-MB-23I } \\
\text { cells) }\end{array}$ & $\begin{array}{l}\text { S.C. xenograft } \\
\text { mouse }\end{array}$ & $\begin{array}{l}\text { NIR fluorescence } \\
\text { imaging }\end{array}$ & $\begin{array}{l}\text { The fluorescence signal in receptor-positive } \\
\text { tumor was distinguishable from the normal } \\
\text { tissues at } 2 \mathrm{~h} \text { post injection, reached peak } \\
\text { intensity at } 10 \mathrm{~h} \text { post injection, and was still } \\
\text { detectable at } 96 \mathrm{~h}\end{array}$ & 107 \\
\hline $\begin{array}{l}\text { Stabilizing ligand: BSA } \\
\text { Targeting ligand: FA } \\
\text { and HA }\end{array}$ & $\begin{array}{l}\text { Liver cancer } \\
\text { (HepG2 cells) } \\
\text { Adenocarcinoma } \\
\text { (A549 cells) }\end{array}$ & $\begin{array}{l}\text { S.C. xenograft } \\
\text { mouse }\end{array}$ & $\begin{array}{l}\text { NIR fluorescence } \\
\text { imaging }\end{array}$ & $\begin{array}{l}\text { The strong fluorescence was observed at } \\
\text { the tumor sites derived from the selectively } \\
\text { accumulated targeted AuNPs, demonstrating a } \\
\text { promising probe for the cancer diagnosis }\end{array}$ & 108 \\
\hline $\begin{array}{l}\text { Stabilizing ligand: BSA } \\
\text { Nuclear imaging moiety: } \\
\text { Hoechst }\end{array}$ & $\begin{array}{l}\text { Pancreatic tumor } \\
\text { (MiaPaca-2 cells) }\end{array}$ & $\begin{array}{l}\text { S.C. xenograft } \\
\text { mouse }\end{array}$ & $\begin{array}{l}\text { Maestro }{ }^{\mathrm{TM}} 2 \text { in vivo } \\
\text { imaging system }\end{array}$ & $\begin{array}{l}\text { The in vivo imaging was performed via blue and } \\
\text { red channels which displayed the accumulation } \\
\text { of Hoechst-AuNCs mainly in the tumor and } \\
\text { partly in the liver and kidneys }\end{array}$ & 109 \\
\hline $\begin{array}{l}\text { Stabilizing ligand: PEG } \\
\text { Emitter: }{ }^{64} \mathrm{Cu}\end{array}$ & $\begin{array}{l}\text { Prostate cancer } \\
\text { (PC3 cells) }\end{array}$ & $\begin{array}{l}\text { S.C. xenograft } \\
\text { mouse }\end{array}$ & $\mathrm{PET} / \mathrm{CT}$ & $\begin{array}{l}\text { PET/CT results demonstrated the } \\
\text { heterogeneous intratumoral distribution of } \\
{ }^{64} \mathrm{CuAuNCs}-\mathrm{PEG} 350 \text { and }{ }^{64} \mathrm{CuAuNCs}-\mathrm{PEG} 1000\end{array}$ & 110 \\
\hline Emitter: ${ }^{64} \mathrm{Cu}$ & $\begin{array}{l}\text { Glioblastoma } \\
\text { (U87 MG cells) }\end{array}$ & $\begin{array}{l}\text { S.C. xenograft } \\
\text { mouse }\end{array}$ & $\begin{array}{l}\text { PET } \\
\text { IVIS }^{\circledR} \text { imaging system }\end{array}$ & $\begin{array}{l}{ }^{64} \mathrm{Cu} \text {-dopped AuNCs showed satisfactory } \\
\text { synergistic dual-modality PET and self- } \\
\text { illuminating NIR tumor imaging }\end{array}$ & 111 \\
\hline $\begin{array}{l}\text { Stabilizing ligand: BSA } \\
\text { Emitter: } \mathrm{Gd}^{3+}\end{array}$ & $\begin{array}{l}\text { Breast cancer } \\
\text { (MCF-7 cells) }\end{array}$ & $\begin{array}{l}\text { S.C. xenograft } \\
\text { mouse }\end{array}$ & $\begin{array}{l}\mathrm{CT} \\
\text { NIR fluorescence } \\
\text { imaging } \\
\text { MRI }\end{array}$ & $\begin{array}{l}\text { The hybrid gold-gadolinium nanoclusters } \\
\text { provided a promising nanoprobe for cancer- } \\
\text { targeted imaging and diagnosis in vivo }\end{array}$ & 112 \\
\hline $\begin{array}{l}\text { Stabilizing ligand: } \\
\text { hairpin-DNA }\end{array}$ & $\begin{array}{l}\text { Melanoma } \\
\text { (MI4 cells) }\end{array}$ & $\begin{array}{l}\text { S.C. xenograft } \\
\text { mouse }\end{array}$ & $\begin{array}{l}\text { NIR fluorescence } \\
\text { imaging }\end{array}$ & $\begin{array}{l}\text { The hairpin-DNA-modified } \mathrm{NaYF}_{4} @ \mathrm{SiO}_{4}-\mathrm{Au} \\
\text { promoted simultaneous deep tissue imaging } \\
\text { and drug molecule release when combining } \\
\text { single-band anti-stokes NIR emission and the } \\
\text { photothermal effect }\end{array}$ & 113 \\
\hline $\begin{array}{l}\mathrm{pH} \text {-sensitive ligand: } \\
\text { azide and alkyne } \\
\text { functionalities }\end{array}$ & $\begin{array}{l}\text { Glioma } \\
\text { (U87MG cells) }\end{array}$ & $\begin{array}{l}\text { Orthotopic } \\
\text { xenograft } \\
\text { mouse }\end{array}$ & $\begin{array}{l}\text { MRI and SERS } \\
\text { imaging }\end{array}$ & $\begin{array}{l}\text { Multifunctional AuNPs could not only } \\
\text { preoperatively define orthotopic glioblastoma } \\
\text { xenografts by MRI with high sensitivity and } \\
\text { durability in vivo but also intraoperatively } \\
\text { guide tumor excision with the assistance of a } \\
\text { handheld Raman scanner }\end{array}$ & 114 \\
\hline \multicolumn{6}{|l|}{ Hollow AuNPs } \\
\hline Stabilizing ligand: PEG & $\begin{array}{l}\text { Adenocarcinoma } \\
\text { (A549 cells) }\end{array}$ & $\begin{array}{l}\text { S.C. xenograft } \\
\text { mouse }\end{array}$ & CT & $\begin{array}{l}\text { The attenuation coefficient of hollow } \\
\text { AuNPs is } 5.3 \text { times higher than that of the } \\
\text { iodine-based contrast agent at the same } \\
\text { concentration, demonstrating the potential of } \\
\text { hollow AuNPs for CT imaging }\end{array}$ & 115 \\
\hline $\begin{array}{l}\text { Stabilizing ligand: PEG } \\
\text { Targeting ligand: RGD } \\
\text { Emitter: }{ }^{64} \mathrm{Cu}\end{array}$ & $\begin{array}{l}\text { Liver carcinoma } \\
\text { VX2 tumor) }\end{array}$ & $\begin{array}{l}\text { Orthotopic } \\
\text { allograft rabbit }\end{array}$ & $\mathrm{PET} / \mathrm{CT}$ & $\begin{array}{l}\text { PET/CT images showed that the }{ }^{64} \mathrm{Cu}-\mathrm{RGD} \text { - } \\
\text { PEG-HAuNS showed higher tumor uptake than } \\
\text { control groups at } 24 \mathrm{~h} \text { post injection }\end{array}$ & 116 \\
\hline $\begin{array}{l}\text { Stabilizing ligand: PEG } \\
\text { Targeting ligand: RGD } \\
\text { Emitter: }{ }^{64} \mathrm{Cu} \\
\text { Gold nanostars }\end{array}$ & $\begin{array}{l}\text { Glioblastoma } \\
\text { (U87 cells) }\end{array}$ & $\begin{array}{l}\text { Orthotopic } \\
\text { xenograft } \\
\text { mouse }\end{array}$ & $\begin{array}{l}\mathrm{PET} / \mathrm{CT} \\
\mathrm{PAI}\end{array}$ & $\begin{array}{l}\text { The dual-modality PAI and PET/CT imaging } \\
\text { provided a promising targeted AuNP-mediated } \\
\text { glioma therapy }\end{array}$ & 117 \\
\hline $\begin{array}{l}\text { Stabilizing ligand: PEG } \\
\text { Raman reporter: } \\
\text { p-mercaptobenzoic acid }\end{array}$ & $\begin{array}{l}\text { Primary soft-tissue } \\
\text { sarcomas }\end{array}$ & $\begin{array}{l}\text { Transgenic } \\
\text { mouse }\end{array}$ & $\begin{array}{l}\text { CT } \\
\text { Two-photon } \\
\text { luminescence } \\
\text { imaging }\end{array}$ & $\begin{array}{l}\text { The CT and optical results showed that } 30 \mathrm{~nm} \\
\text { nanostars have higher tumor uptake, as well as } \\
\text { deeper penetration into tumor interstitial space } \\
\text { compared with } 60 \mathrm{~nm} \text { counterparts }\end{array}$ & 118 \\
\hline
\end{tabular}


Table I (Continued)

\begin{tabular}{|c|c|c|c|c|c|}
\hline Functional ligand & Cancer type & In vivo model & Imaging technique & Comment & Reference \\
\hline Stabilizing ligand: PEG & $\begin{array}{l}\text { Breast cancer } \\
(4 T I \text { cells })\end{array}$ & $\begin{array}{l}\text { S.C. allograft } \\
\text { mouse }\end{array}$ & $\mathrm{PAI}$ & $\begin{array}{l}\text { Novel } \mathrm{Fe}_{2} \mathrm{O}_{3} @ \mathrm{Au} \text { core/shell magnetic gold } \\
\text { nanoflowers were synthesized through } \\
\text { interactive growth of } \mathrm{Au} \text { on } \mathrm{Fe}_{2} \mathrm{O}_{3} \mathrm{NPs} \text {. The } \\
\text { nanoflowers exhibited remarkable SERS } \\
\text { enhancement }\end{array}$ & 119 \\
\hline Emitter: $\mathrm{Gd}^{3+}$ & $\begin{array}{l}\text { Adenocarcinoma } \\
\text { (A549 cells) }\end{array}$ & $\begin{array}{l}\text { S.C. xenograft } \\
\text { mouse }\end{array}$ & $\begin{array}{l}\text { MRI, CT, and NIR } \\
\text { fluorescence imaging }\end{array}$ & $\begin{array}{l}\text { The existence of } \mathrm{Gd}^{3+} \text { ions on } \mathrm{GNCN} \text { s exhibits } \\
\text { significant luminescence intensity enhancement } \\
\text { for NIR fluorescence imaging, high } \mathrm{X} \text {-ray } \\
\text { attenuation for CT imaging, and reasonable rI } \\
\text { relaxivity for MRI }\end{array}$ & 120 \\
\hline $\begin{array}{l}\text { SERS labeling ligand: } \\
\text { DTNB }\end{array}$ & $\begin{array}{l}\text { Ovarian cancer } \\
\text { (SKOV3) }\end{array}$ & $\begin{array}{l}\text { S.C. xenograft } \\
\text { mouse }\end{array}$ & Raman spectroscopy & $\begin{array}{l}\text { The SERS Au nanostars were developed as } \\
\text { a highly sensitive contrast agent for tumor } \\
\text { detection in xenografted mice }\end{array}$ & 121 \\
\hline \multicolumn{6}{|l|}{ Gold nanocages } \\
\hline $\begin{array}{l}\text { Stabilizing ligand: PEG } \\
\text { Emitter: }{ }^{64} \mathrm{Cu}\end{array}$ & $\begin{array}{l}\text { Breast cancer } \\
\text { (EMT-6 cells) }\end{array}$ & $\begin{array}{l}\text { S.C. xenograft } \\
\text { mouse }\end{array}$ & $\mathrm{PET} / \mathrm{CT}$ & $\begin{array}{l}\text { PET/CT images clearly showed rapid localization } \\
\text { of the }{ }^{64} \mathrm{Cu}-\mathrm{PEG}-\mathrm{Au} \text { nanocages in tumor at } \mathrm{I} \mathrm{h} \\
\text { post injection with the administration of a trace } \\
\text { amount }\end{array}$ & 122 \\
\hline \multicolumn{6}{|l|}{ Gold nanoprisms } \\
\hline Stabilizing ligand: PEG & $\begin{array}{l}\text { Colorectal cancer } \\
\text { (HT-29 cells) }\end{array}$ & $\begin{array}{l}\text { S.C. xenograft } \\
\text { mouse }\end{array}$ & $\mathrm{PAI}$ & $\begin{array}{l}\text { PEGylated Au nanoprisms showed the capacity } \\
\text { to penetrate tumors and provided a high- } \\
\text { resolution signal amplifier for optoacoustic } \\
\text { imaging }\end{array}$ & 123 \\
\hline \multicolumn{6}{|l|}{ Gold nanotripods } \\
\hline $\begin{array}{l}\text { Stabilizing ligand: PEG } \\
\text { Targeting ligand: RGD }\end{array}$ & $\begin{array}{l}\text { Glioblastoma (U87 } \\
\text { MG cells) }\end{array}$ & $\begin{array}{l}\text { S.C. xenograft } \\
\text { mouse }\end{array}$ & $\mathrm{PAl}$ & $\begin{array}{l}\text { i.v. injection of RGD-conjugated Au-tripods } \\
\text { showed PAI contrasts in tumors up to threefold } \\
\text { higher than for the blocking group (coinjection } \\
\text { with RGD) }\end{array}$ & 124 \\
\hline
\end{tabular}

Abbreviations: AIE, aggregation-induced emission; AuNPs, gold NPs; BSA, bovine serum albumin; CET, cetuximab; CT, computed tomography; DAPTA, D-Alalpeptide T-amide; DTNB, 5,5-dithio-bis-(2-nitrobenzoic acid); FA, folic acid; GC, glycol chitosan; GNRs, gold nanorods; HA, hyaluronic acid; ICG, indocyanine green; i.v., intravenous; LbL, layer-by-layer; MMP, matrix metalloproteinase; MPNA, magnetoplasmonic nanoassembly; MRI, magnetic resonance imaging; NGAL, neutrophil gelatinaseassociated lipocalin; NIR, near-infrared; NPs, nanoparticles; PAI, photoacoustic imaging; PB, Prussian blue; PEG, polyethylene glycol; PEI, polyethylenimine; PET, positron emission tomography; PLGA, poly(lactic-co-glycolic acid); PPAA, plasma-polymerized allylamine shell; PTT, photothermal therapy; SPECT, single-photon emission CT; S.C., subcutaneous; SERS, surface-enhanced Raman spectroscopy; TAT, transactivator of transcription; TNBC, triple-negative breast cancer.

of AuNPs, functional ligands, cancer types, in vivo animal models, imaging techniques, and end point comments.

\section{AuNPs for cancer phototherapy PTT}

In addition to the aforementioned enhanced light scattering (referred as a radiative property) useful for optical imaging, AuNPs can also convert the absorbed light into heat via a series of nonradiative processes. ${ }^{86,125-129}$ Two main processes occur based on the heat energy contents: 1) the heat from the energy transformation is passed to the surrounding medium via the phonon-phonon relaxation within $\sim 100$ ps and 2) a competitive process takes place between the heating by the electrons and the cooling by the surrounding medium, and when the heating rate is much faster than the cooling rate, AuNPs are melted in hundreds of femtoseconds. ${ }^{125-128}$ To facilitate cancer PTT, the first process has to dominate, and therefore, continuous-wave (CW) lasers that overlap maximally with the AuNP SPR absorption band need to be utilized. ${ }^{130,131}$
PTT can be achieved using gold nanospheres under the stimulation of pulsed or CW visible lasers due to the SPR absorption in the visible region, whereby such treatment is suitable for shallow tumors (ie, skin cancer). ${ }^{132,133}$ Recently, antibody-targeted gold nanospheres were developed to specifically target the EGFR on squalors carcinoma cells, and following stimulation by single 10 ns laser pulses at visible wavelengths, the resultant AuNPs generated intracellular photothermal micro bubbles and induced PTT for tumor inhibition in a subcutaneous cancer model. ${ }^{134}$

To treat tumors under the skin, NIR-active PTT is favorable as the light can penetrate more deeply due to the minimal absorption of the hemoglobin and water in tissues in this spectral region. Therefore, it is important to tune the SPR absorption of AuNPs to the NIR region by means of altering the shape, morphology, and structure, as described in the "Optical characteristics of AuNPs" section.

Recently, RGD-conjugated dendrite-modified GNRs (RGD-diners) were developed by $\mathrm{Li}$ et $\mathrm{al}^{135}$ for selective 
tumor targeting and PTT in xenografted mice. At $6 \mathrm{~h}$ after intravenous injection, the resultant AuNPs accumulated inside tumor tissues via targeting the $\alpha_{\mathrm{v}} \beta_{3}$ overexpressed on cancer cells, and when it was irradiated with a NIR laser with a wavelength of $808 \mathrm{~nm}$ at a power density of $24 \mathrm{~W} / \mathrm{cm}^{2}$ ( $\sim 0.5 \mathrm{~cm}$ diameter illuminated region), tumor growth was significantly reduced by RGD-diners in comparison with the control groups.

In addition, polymeric per fluorocarbon Nan capsules were prepared using the oil-in-water emulsion method, followed by the addition of PEGylated gold nanoshells on the surface. ${ }^{136}$ These resultant Nan capsules could not only enhance the contrast for ultrasound/CT imaging but also function as photo-absorbers for NIR-active PTT in xenografted mice. ${ }^{136}$

Recently, Ayala-Orozco et a ${ }^{137}$ have developed novel gold nanomatryoshkas composed of concentric gold-silica-gold layers and PEG-stabilizing ligands, for PTT of TNBC. In comparison with $\sim 150 \mathrm{~nm}$ conventional silica-Au nanoshells, the resultant gold nanomatryoshkas $(<100 \mathrm{~nm})$ facilitated higher accumulation in tumors due to better penetration of smaller AuNPs into tissue via the EPR effect. Under irradiation with a $\mathrm{CW}$ laser emitting $3 \mathrm{~W} / \mathrm{cm}^{2}$ at a wavelength of $808 \mathrm{~nm}$ ( $\sim 1.2 \mathrm{~cm}$ diameter illuminated region), the survival rate of an advanced TNBC model with $>1,000 \mathrm{~mm}^{3}$ tumors was significantly improved by gold nanomatryoshkas relative to conventional silica-Au nanoshells. ${ }^{137}$

\section{Photodynamic therapy (PDT)}

When photosensitizers are stimulated under the light of specific wavelengths, they convert the surrounding oxygen into toxic reactive oxygen species (ie, singlet oxygen) that may destroy malignant cells in surrounding proximity, which is now known as cancer PDT. ${ }^{138}$ However, most of the organic photosensitizers are only activated by UV and visible lights, which have poor tissue penetration and therefore are limited to the treatment of surface tumors. ${ }^{139}$ In addition, organic photosensitizers possess low molar extinction coefficients and therefore can undergo photobleaching and enzymatic degradation. ${ }^{139}$ In contrast, metal nanostructures (ie, gold, silver, and platinum) can overcome these limitations, as they possess 5-6 orders of molar extinction coefficients, better photostability, and enhanced resistant to enzymatic degradation. ${ }^{140}$

To tackle the treatment of deeply buried tumors, AuNPs that are able to exert PDT upon NIR light activation have recently been developed. For example, lipid-coated gold nanocages were produced to activate PTT and PDT simultaneously in melanoma-xenografted mice. ${ }^{141}$ Following direct injection of lipid-coated gold nanocages into the tumor site, a $980 \mathrm{~nm} \mathrm{CW}$ laser $\left(150 \mathrm{~mW} / \mathrm{cm}^{2} ; 10 \mathrm{~min}\right)$ was used to stimulate AuNPs, which raised temperature $\left(\sim 10^{\circ} \mathrm{C}\right)$ and generated the singlet oxygen. As a result, this treatment significantly inhibited tumor growth in comparison with an irradiation of the $808 \mathrm{~nm}$ diode laser $\left(150 \mathrm{~mW} / \mathrm{cm}^{2} ; 12 \mathrm{~min}\right.$; only PTT was induced in this wavelength).

In addition, a variety of organic photosensitizers with NIR-active property can also be incorporated into AuNPs for PDT with a low dose of organic photosensitizers and short exposure of laser irradiation. For example, indocyanine green (ICG, a medical diagnostic agent) is used to produce singlet oxygen for PDT. ${ }^{142}$ Recently, GNR/ICG-loaded chitosan nanospheres (CS-AuNR-ICG NSs) were prepared by the nonsolvent counterion complexation method and electrostatic interaction. ${ }^{142}$ The CS-AuNR-ICG NSs could effectively load ICG and protect it from rapid hydrolysis. Results of in vivo NIR fluorescence imaging and biodistribution showed that these Au-chitosan NPs could be specifically delivered to the tumor site. Under an $808 \mathrm{~nm}$ laser, CS-AuNR-ICG NSs simultaneously produced hyperthermia and reactive oxygen species, which achieved complete inhibition of tumor growth in xenografted mice. Compared with PTT (GNRs) or PDT (ICG) alone, the combined therapy showed a significantly improved therapeutic efficacy. ${ }^{142}$

In addition, selected examples of AuNP-based cancer phototherapy are provided in Table 2 , including the types of AuNPs, functional ligands, cancer types, in vivo animal models, laser types, and end point comments.

\section{Barriers to the translation of AuNPs for cancer theranostics}

Although local administration of therapeutics has demonstrated significant potential for the treatment of shallow cancers (ie, skin cancer and head and neck cancer) due to the ease of access, many diseases (eg, metastatic cancers) still require systemic administration of therapeutic agents into the bloodstream. In this section, major challenges, namely, 1) instability in the blood circulation, 2) targeting to specific cells of interest, and 3) activation in response to the tumor microenvironment (TME),${ }^{174}$ for systemic AuNP delivery in terms of cancer diagnosis and phototherapeutics are discussed (Figure 2).

\section{Blood circulation}

It has been reported, following intravenous administration, that rapid glomerular filtration occurs in the case 
Table 2 A summary of studies on the in vivo use of gold nanocomplexes in systemic cancer PTT and PDT

\begin{tabular}{|c|c|c|c|c|c|c|}
\hline AuNP type & Functional ligand & Cancer type & In vivo model & Laser & Comment & Reference \\
\hline \multicolumn{7}{|l|}{ PTT } \\
\hline Au nanorods & Stabilizing ligand: PEG & $\begin{array}{l}\text { Melanoma (MDA- } \\
\text { MB-435 cells) }\end{array}$ & $\begin{array}{l}\text { S.C. xenograft } \\
\text { mouse }\end{array}$ & $\begin{array}{l}810 \mathrm{~nm} \text { laser } \\
2 \mathrm{~W} / \mathrm{cm}^{2} \\
5 \mathrm{~min}\end{array}$ & $\begin{array}{l}\text { A single i.v. injection of PEG-Au nanorods } \\
\text { enabled destruction of the irradiated } \\
\text { human xenograft tumors in mice }\end{array}$ & 143 \\
\hline Au nanorods & $\begin{array}{l}\text { Stabilizing ligand: PEG } \\
\text { Targeting ligand: } \\
\text { RGD }\end{array}$ & $\begin{array}{l}\text { Glioblastoma (U87 } \\
\text { MG cells) }\end{array}$ & $\begin{array}{l}\text { S.C. xenograft } \\
\text { mouse }\end{array}$ & $\begin{array}{l}808 \mathrm{~nm} \text { laser } \\
\mathrm{I} \mathrm{W} / \mathrm{cm}^{2} \\
10 \mathrm{~min}\end{array}$ & $\begin{array}{l}\text { Au nanorods showed high tumor-targeting } \\
\text { ability via receptor-mediated pathway and } \\
\text { were successfully used for PTT }\end{array}$ & 144 \\
\hline Au nanorods & $\begin{array}{l}\text { Coating material: } \\
\text { silica }\end{array}$ & $\begin{array}{l}\text { Breast cancer }(4 \mathrm{TI} \\
\text { cells) }\end{array}$ & $\begin{array}{l}\text { S.C. allograft } \\
\text { mouse }\end{array}$ & $\begin{array}{l}808 \mathrm{~nm} \text { laser } \\
4 \mathrm{~W} / \mathrm{cm}^{2} \\
10 \mathrm{~min}\end{array}$ & $\begin{array}{l}\text { When Au nanorods were stimulated } \\
\text { with the NIR laser, DOX was released } \\
\text { for synergistic therapeutic effect in } \\
\text { combination with PTT }\end{array}$ & 145 \\
\hline Au nanorods & $\begin{array}{l}\text { Stabilizing ligand: PEG } \\
\text { and dendrimers }\end{array}$ & $\begin{array}{l}\text { Colon carcinoma } \\
\text { ( } 26 \text { cells) }\end{array}$ & $\begin{array}{l}\text { S.C. allograft } \\
\text { mouse }\end{array}$ & $\begin{array}{l}808 \mathrm{~nm} \text { laser } \\
0.24 \mathrm{~W} / \mathrm{cm}^{2} \\
10 \mathrm{~min}\end{array}$ & $\begin{array}{l}\text { The combined photothermal-chemo } \\
\text { treatment using AuNPs containing DOX } \\
\text { for synergistic PPT and chemotherapy } \\
\text { exhibited higher therapeutic efficacy than } \\
\text { either single treatment alone }\end{array}$ & 146 \\
\hline Au nanorods & $\begin{array}{l}\text { Coating materials: } \\
\text { PVP and } \mathrm{AgNO}_{3} \\
\text { Targeting ligand: } \\
\text { aptamer }\end{array}$ & $\begin{array}{l}\text { Adenocarcinoma } \\
\text { (A549 cells) }\end{array}$ & $\begin{array}{l}\text { S.C. xenograft } \\
\text { mouse }\end{array}$ & $\begin{array}{l}980 \mathrm{~nm} \text { laser } \\
0.84 \mathrm{~W} / \mathrm{cm}^{2} \\
5 \mathrm{~min}\end{array}$ & $\begin{array}{l}\text { The resultant AuNPs specifically } \\
\text { accumulated into tumor tissues and } \\
\text { induced PTT for dramatically stronger } \\
\text { antitumor effect upon NIR laser irradiation }\end{array}$ & 147 \\
\hline Au nanorods & $\begin{array}{l}\text { Au nanorods } \\
\text { encapsulated in } \\
\text { CHI/sodium ALG } \\
\text { microcapsules }\end{array}$ & $\begin{array}{l}\text { Breast cancer } \\
(4 \mathrm{TI} \text { cells) }\end{array}$ & $\begin{array}{l}\text { S.C. allograft } \\
\text { mouse }\end{array}$ & $\begin{array}{l}808 \mathrm{~nm} \text { laser } \\
3.83 \mathrm{~J} / \mathrm{cm}^{2} \\
5 \mathrm{~min}\end{array}$ & $\begin{array}{l}\text { Self-assembled Au nanorods in bilayer- } \\
\text { modified microcapsules localized at tumor } \\
\text { sites, generated vapor bubbles under NIR } \\
\text { exposure, and subsequently damaged } \\
\text { tumor tissues }\end{array}$ & 148 \\
\hline Au nanorods & $\begin{array}{l}\text { Stabilizing ligand: PEG } \\
\text { Targeting ligand: } \\
\text { antibody for } \\
\text { anaerobic bacteria } \\
\text { (C. difficile) }\end{array}$ & $\begin{array}{l}\text { Adenocarcinoma } \\
\text { (A549 cells) }\end{array}$ & $\begin{array}{l}\text { S.C. xenograft } \\
\text { mouse }\end{array}$ & $\begin{array}{l}808 \mathrm{~nm} \text { laser } \\
0.5 \mathrm{~W} / \mathrm{cm}^{2} \\
10 \mathrm{~min}\end{array}$ & $\begin{array}{l}\text { The } C \text {. difficile spores was i.v. injected } \\
\text { for } 2 \text { days, followed by the injection of } \\
\text { antibody-Au nanorods to specifically target } \\
\text { the germination of the } C \text {. difficile spores } \\
\text { in tumor tissues (low level of oxygen } \\
\text { microenvironment). Under the NIR laser, } \\
\text { antibody-Au nanorods completely inhibited } \\
\text { tumor growth }\end{array}$ & 149 \\
\hline Au nanorods & $\begin{array}{l}\text { Stabilizing ligand: } \\
\text { dendrimer }\end{array}$ & $\begin{array}{l}\text { Non-small-cell lung } \\
\text { cancer (PC-9 cells) }\end{array}$ & $\begin{array}{l}\text { S.C. xenograft } \\
\text { mouse }\end{array}$ & $\begin{array}{l}808 \mathrm{~nm} \text { laser } \\
3.6 \mathrm{~W} / \mathrm{cm}^{2} \\
8 \mathrm{~min}\end{array}$ & $\begin{array}{l}\text { Dendrimer-stabilized Au nanorods } \\
\text { (DSAuNRs, sub- } 10 \mathrm{~nm} \text { in length) showed } \\
\text { significantly enhanced absorption in the } \\
\text { NIR region compared with dendrimer- } \\
\text { stabilized Au nanospheres. The tumor } \\
\text { growth was significantly retarded by the } \\
\text { photothermal efficiency of DSAuNRs }\end{array}$ & 150 \\
\hline Au nanorods & $\begin{array}{l}\text { Coating material: } \\
\text { silica } \\
\text { Targeting ligand: } \\
\text { antibody for CXCR4 }\end{array}$ & $\begin{array}{l}\text { Gastric cancer } \\
\text { (MGC803 cells) }\end{array}$ & $\begin{array}{l}\text { S.C. xenograft } \\
\text { mouse }\end{array}$ & $\begin{array}{l}808 \mathrm{~nm} \text { laser } \\
1.5 \mathrm{~W} / \mathrm{cm}^{2} \\
3 \mathrm{~min}\end{array}$ & $\begin{array}{l}\text { iPS cells were transfected with the } \\
\text { resulted AuNRs@SiO2@CXCR4 } \\
\text { via receptor-mediated pathway. The } \\
\text { transfected iPS cells were homing to } \\
\text { tumor tissues, and the tumor growth } \\
\text { was significantly slowed down by the } \\
\text { photothermal efficiency of AuNRs@ } \\
\text { SiO2@CXCR4 }\end{array}$ & $15 \mid$ \\
\hline Au nanoshells & $\begin{array}{l}\text { Multilayered AuNPs } \\
\text { with silica and gold, } \\
\text { also termed Au } \\
\text { nanomatryoshkas }\end{array}$ & $\begin{array}{l}\text { Breast cancer } \\
\text { (MDA-MB-23 I cells) }\end{array}$ & $\begin{array}{l}\text { Orthotopic } \\
\text { xenograft mouse }\end{array}$ & $\begin{array}{l}810 \mathrm{~nm} \text { laser } \\
2 \mathrm{~W} / \mathrm{cm}^{2} \\
5 \mathrm{~min}\end{array}$ & $\begin{array}{l}\text { Au nanomatryoshkas exhibited improved } \\
\text { PTT efficacy when compared with } \\
\text { conventional gold nanoshells }\end{array}$ & 152 \\
\hline Au nanoshells & Stabilizing ligand: PEG & $\begin{array}{l}\text { Breast cancer } \\
(4 \mathrm{TI} \text { cells) }\end{array}$ & $\begin{array}{l}\text { S.C. allograft } \\
\text { mouse }\end{array}$ & $\begin{array}{l}808 \mathrm{~nm} \text { laser } \\
1 \mathrm{~W} / \mathrm{cm}^{2} \\
10 \mathrm{~min}\end{array}$ & $\begin{array}{l}\text { In combination with chemotherapeutics, } \\
\text { the resultant Au nanoshells achieved } \\
\text { complete destruction of the tumors at a } \\
\text { low laser irradiation without weight loss } \\
\text { or recurrence of tumors }\end{array}$ & 153 \\
\hline
\end{tabular}


Table 2 (Continued)

\begin{tabular}{|c|c|c|c|c|c|c|}
\hline AuNP type & Functional ligand & Cancer type & In vivo model & Laser & Comment & Reference \\
\hline Au nanoshells & $\begin{array}{l}\text { Stabilizing ligand: PEG } \\
\text { Inner core: PLGA } \\
\text { NPs }\end{array}$ & $\begin{array}{l}\text { Glioblastoma (U87 } \\
\text { MG cells) }\end{array}$ & $\begin{array}{l}\text { S.C. xenograft } \\
\text { mouse }\end{array}$ & $\begin{array}{l}808 \mathrm{~nm} \text { laser } \\
1.5 \mathrm{~W} / \mathrm{cm}^{2} \\
1.5 \mathrm{~min}\end{array}$ & $\begin{array}{l}\text { The temperature of tumor treated with } \\
\text { the resultant Au nanoshells was rapidly } \\
\text { increased to } 46.6^{\circ} \mathrm{C} \text {, which released } \\
\text { DOX for synergistic therapeutic effect in } \\
\text { combination with PTT }\end{array}$ & 154 \\
\hline Au nanoshells & $\begin{array}{l}\text { Stabilizing ligand: PEG } \\
\text { Inner core: PEI-PAsp } \\
\text { (DIP/MEA) NPs }\end{array}$ & $\begin{array}{l}\text { Liver cancer } \\
\text { Bel-7402 cells }\end{array}$ & $\begin{array}{l}\text { S.C. xenograft } \\
\text { mouse }\end{array}$ & $\begin{array}{l}808 \mathrm{~nm} \text { laser } \\
1.5 \mathrm{~W} / \mathrm{cm}^{2} \\
2 \mathrm{~min}\end{array}$ & $\begin{array}{l}\text { A polymeric vesicle encapsulating DOX } \\
\text { was prepared and then decorated with a } \\
\text { gold layer using a modified method of in } \\
\text { situ gold seed growth. The NIR light energy } \\
\text { was converted into heat, which killed } \\
\text { cancer cells in the vicinity and induced } \\
\text { the rupture of nanoshell to release DOX } \\
\text { inside tumor }\end{array}$ & 155 \\
\hline Au nanoshells & $\begin{array}{l}\text { Stabilizing coating: } \\
\text { MPCMs } \\
\text { Inner core: silica }\end{array}$ & $\begin{array}{l}\text { Breast cancer } \\
(4 \mathrm{TI} \text { cells })\end{array}$ & $\begin{array}{l}\text { S.C. allograft } \\
\text { mouse }\end{array}$ & 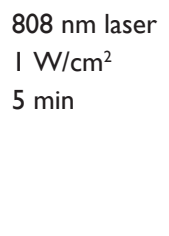 & $\begin{array}{l}\text { MPCM-coated Au nanoshells presented } \\
\text { longer blood circulation and tumor } \\
\text { accumulation in a xenograft mouse model } \\
\text { of breast cancer. Tumor growth was } \\
\text { significantly slowed down by irradiation of } \\
\text { NIR laser }\end{array}$ & 156 \\
\hline Au nanostars & $\begin{array}{l}\text { Stabilizing ligand: PEG } \\
\text { Targeting ligand: } \\
\text { RGD }\end{array}$ & $\begin{array}{l}\text { Glioblastoma } \\
\text { (U87 MG cells) }\end{array}$ & $\begin{array}{l}\text { S.C. xenograft } \\
\text { mouse }\end{array}$ & $\begin{array}{l}790 \mathrm{~nm} \text { laser } \\
1 \mathrm{~W} / \mathrm{cm}^{2} \\
10 \mathrm{~min}\end{array}$ & $\begin{array}{l}\text { RGD-Au nanostars were designed to } \\
\text { specifically target overexpressed integrin } \\
\alpha_{v} \beta_{3} \text { on tumor neovasculature, enabling } \\
\text { highly sensitive PTT }\end{array}$ & 157 \\
\hline Au nanostars & $\begin{array}{l}\text { Surface coating: } \\
\text { organosilica }\end{array}$ & $\begin{array}{l}\text { Breast cancer } \\
\text { (MDA-MB-23I cells) }\end{array}$ & $\begin{array}{l}\text { S.C. xenograft } \\
\text { mouse }\end{array}$ & $\begin{array}{l}808 \mathrm{~nm} \text { laser } \\
0.5 \mathrm{~W} / \mathrm{cm}^{2} \\
5 \mathrm{~min}\end{array}$ & $\begin{array}{l}\text { In } 5 \text { min of irradiation, the temperature } \\
\text { at the tumor region of mice treated with } \\
\text { Au nanostars increased remarkably to } \\
\text { about } 57^{\circ} \mathrm{C}\end{array}$ & 158 \\
\hline Au nanocages & Targeting ligand: HA & $\begin{array}{l}\text { Breast cancer } \\
\text { (MDA-MB-23I cells) }\end{array}$ & $\begin{array}{l}\text { S.C. xenograft } \\
\text { mouse }\end{array}$ & $\begin{array}{l}808 \mathrm{~nm} \text { laser } \\
1 \mathrm{~W} / \mathrm{cm}^{2} \\
5 \mathrm{~min}\end{array}$ & $\begin{array}{l}\text { HA-coated Au nanocages accumulated } \\
\text { inside tumor tissues via HA-CD } 44 \\
\text { interaction. Under the NIR stimulation, } \\
\text { HA-coated Au nanocages significantly } \\
\text { slowed down the tumor growth. In } \\
\text { addition, a complete tumor inhibition } \\
\text { was achieved when combined with } \\
\text { chemotherapy }\end{array}$ & 159 \\
\hline Au nanocages & $\begin{array}{l}\text { Gold surface was } \\
\text { coated with PVP and } \\
\text { RBC membranes }\end{array}$ & $\begin{array}{l}\text { Breast cancer } \\
(4 \mathrm{TI} \text { cells) }\end{array}$ & $\begin{array}{l}\text { S.C. allograft } \\
\text { mouse }\end{array}$ & 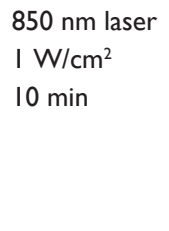 & $\begin{array}{l}\text { RBC-AuNCs exhibited significantly } \\
\text { enhanced in vivo blood retention and } \\
\text { circulation lifetime. With NIR laser, } \\
\text { RBC-AuNCs achieved I00\% survival } \\
\text { of tumor-bearing mice over a span of } \\
45 \text { days }\end{array}$ & 160 \\
\hline $\begin{array}{l}\text { Hollow Au } \\
\text { nanospheres }\end{array}$ & $\begin{array}{l}\text { Stabilizing ligand: PEG } \\
\text { Targeting ligand: } \\
\text { a peptide (TNYL) }\end{array}$ & $\begin{array}{l}\text { Ovarian carcinoma } \\
\text { (SKOV3 cells) }\end{array}$ & $\begin{array}{l}\text { S.C. xenograft } \\
\text { mouse }\end{array}$ & $\begin{array}{l}808 \mathrm{~nm} \text { laser } \\
1.5 \mathrm{~W} / \mathrm{cm}^{2} \\
3 \mathrm{~min}\end{array}$ & $\begin{array}{l}\text { Under NIR laser irradiation, the resultant } \\
\text { hollow Au nanospheres induced PTT for } \\
\text { dramatically stronger antitumor effect } \\
\text { against EphB4-positive tumors than EphB4- } \\
\text { negative tumors }\end{array}$ & 161 \\
\hline $\begin{array}{l}\text { Hollow Au } \\
\text { nanospheres }\end{array}$ & $\begin{array}{l}\text { Stabilizing ligand: PVP } \\
\text { and citrate }\end{array}$ & $\begin{array}{l}\text { Ovarian carcinoma } \\
\text { (SKOV3 cells) }\end{array}$ & $\begin{array}{l}\text { S.C. xenograft } \\
\text { mouse }\end{array}$ & $\begin{array}{l}808 \mathrm{~nm} \\
3.0 \mathrm{~W} / \mathrm{cm}^{2} \\
10 \mathrm{~min}\end{array}$ & $\begin{array}{l}\text { The resultant AuNPs exhibited a } \\
\text { significantly enhanced surface plasmon } \\
\text { absorption in the NIR region, inducing } \\
\text { an efficient photothermal conversion and } \\
\text { stronger anticancer ability under NIR laser } \\
\text { irradiation }\end{array}$ & 162 \\
\hline $\begin{array}{l}\mathrm{Au} \\
\text { nanoclusters }\end{array}$ & $\begin{array}{l}\text { A pH-sensitive } \\
\text { ligand inducing Au } \\
\text { nanoclusters in mild } \\
\text { acidic environments }\end{array}$ & $\begin{array}{l}\text { Fibrosarcoma } \\
\text { (HT-I080 cells) }\end{array}$ & $\begin{array}{l}\text { S.C. xenograft } \\
\text { mouse }\end{array}$ & $\begin{array}{l}660 \mathrm{~nm} \text { laser } \\
0.5 \mathrm{~W} / \mathrm{cm}^{2} \\
1 \mathrm{~min}\end{array}$ & $\begin{array}{l}\text { MSCs were first transfected with the } \\
\text { resultant AuNPs. The MSC-AuNPs } \\
\text { showed a } 37 \text {-fold higher tumor-targeting } \\
\text { efficiency and resulted in a significantly } \\
\text { enhanced anticancer effect upon } \\
\text { irradiation }\end{array}$ & 163 \\
\hline
\end{tabular}


Table 2 (Continued)

\begin{tabular}{lllllll}
\hline AuNP type & Functional ligand & Cancer type & In vivo model & Laser & Comment & Reference \\
\hline Au & Stabilizing ligand: PEG & Breast cancer & S.C. allograft & $808 \mathrm{~nm}$ laser & PEGylated AuNPs presented good \\
nanoplates & & (4TI cells) & mouse & $\begin{array}{l}0.5 \mathrm{~W} / \mathrm{cm}^{2} \\
10 \mathrm{~min}\end{array}$ & $\begin{array}{l}\text { biocompatibility, prolonged blood } \\
\text { circulation, and relatively high tumor }\end{array}$ \\
& & & & & $\begin{array}{l}\text { accumulation. The NIR laser irradiation } \\
\text { induced PTT and retarded tumor growth }\end{array}$
\end{tabular}

PDT

nanospheres

Coating materials:

Adenocarcinoma

heparin

Photosensitizer: PhA

Au nanorods Coating materials:

silica and PEG

Photosensitizer: PPIX

Au nanorods AuNPs encapsulated Squamous in Pluronic nanogel carcinoma Photosensitizer: Ce6 (SCC7 cells)

Au nanorods Stabilizing ligand: PEG Melanoma Targeting ligand: FA (BI6F0 cells)

Au nanorods Stabilizing ligand: $\mathrm{CHI}$ Liver cancer Photosensitizer: ICG (H22 cells)

Au nanorods Stabilizing ligand: Oral squamous poly(allylamine carcinoma hydrochloride) Photosensitizer: RB

Au nanorods Endosome disruptive ligand: Tat/HA2 Photosensitizer: Adenocarcinoma $\mathrm{AIPCS}_{4}$
S.C. xenograft

mouse

S.C. xenograft

mouse

S.C. allograft

mouse

S.C. allograft

mouse

S.C. allograft

mouse

A carcinogen

was topically

$1.79 \mathrm{~W} / \mathrm{cm}^{2}$

left cheek pouch $10 \mathrm{~min}$

mucosa

S.C. xenograft

mouse

$20 \mathrm{~J} / \mathrm{cm}^{2}$

$30 \mathrm{~min}$

$670 \mathrm{~nm}$ laser The PDT effects of PhA-H/AuNP

$30 \mathrm{~min} \quad$ comparison with $\mathrm{PhA}$ alone

$532 \mathrm{~nm}$ laser $A$ real-time and specific in vivo SERS and

$25 \mathrm{~mW} / \mathrm{cm}^{2}$ fluorescence detection method using the

15 min resultant AuNPs was applied for tumor detection and subsequent PDT

$655 \mathrm{~nm}$ laser A remarkable synergy for anticancer treatment was observed when PDT

was applied before PTT, both

in vitro and in vivo

$915 \mathrm{~nm} \quad$ GNRs alone can sensitize the formation

168

$130 \mathrm{~mW} / \mathrm{cm}^{2}$ of singlet oxygen and exert dramatic PDT

$15 \mathrm{~min} \quad$ effects on complete destruction of tumors in mice under light excitation

$808 \mathrm{~nm}$ laser The resultant NPs have been successfully

$2 \mathrm{~W} / \mathrm{cm}^{2} \quad$ prepared to facilitate in vivo PDT resulting

$10 \mathrm{~min}$ in abundant ROS produced by ICG under NIR irradiation

$532 \mathrm{~nm} \quad$ The PDT-only treatment achieved a 46.5\%

tumor inhibition rate; when combined with

(n)


PTT effects under NIR laser stimulation, 95.5\% tumor inhibition rate was achieved

$808 \mathrm{~nm}$ laser AuNRs absorbed an SPR wavelength

$400 \mathrm{~mW} / \mathrm{cm}^{2}$ (808 $\left.\mathrm{nm}\right)$ and converted it into

$15 \mathrm{~min}$ and heat, causing the release of $\mathrm{AIPcS}_{4}$.

$680 \mathrm{~nm}$ LED Subsequently, upon illumination at $680 \mathrm{~nm}$,

light the released $\mathrm{AIPcS}_{4}$ transferred the photon

$10 \mathrm{~mW} / \mathrm{cm}^{2}$ energy to oxygen molecules, stimulating

$40 \mathrm{~min} \quad \mathrm{ROS}$ generation to slow down the tumor growth

\begin{tabular}{|c|c|c|c|c|c|}
\hline Au nanocages & $\begin{array}{l}\text { Stabilizing ligand: PEG } \\
\text { Photosensitizer: }\end{array}$ & $\begin{array}{l}\text { Colon cancer } \\
\text { (Colon-26 cells) }\end{array}$ & $\begin{array}{l}\text { S.C. allograft } \\
\text { mouse }\end{array}$ & $\begin{array}{l}665 \mathrm{~nm} \text { laser } \\
75 \mathrm{~mW} / \mathrm{cm}^{2}\end{array}$ & $\begin{array}{l}\text { The tumor growth was suppressed due to } \\
\text { the enhanced phototoxicity of the HPPH- }\end{array}$ \\
\hline & $\mathrm{HPPH}$ & & & $30 \mathrm{~min}$ & Au nanocages under the laser stimulation \\
\hline $\begin{array}{l}\mathrm{Au} \\
\text { nanoclusters }\end{array}$ & $\begin{array}{l}\text { AuNPs encapsulated } \\
\text { in silica } \\
\text { Photosensitizer: Ce6 }\end{array}$ & $\begin{array}{l}\text { Melanoma (MDA- } \\
\text { MB-435 cells) }\end{array}$ & $\begin{array}{l}\text { S.C. xenograft } \\
\text { mouse }\end{array}$ & $\begin{array}{l}671 \mathrm{~nm} \text { laser } \\
100 \mathrm{~mW} / \mathrm{cm}^{2} \\
10 \mathrm{~min}\end{array}$ & $\begin{array}{l}\text { The resultant AuNCs@SiO }-\mathrm{Ce} 6 \\
\text { completely inhibited tumor growth in mice } \\
\text { due to PDT effects when compared with } \\
\text { Ce6 alone and AuNCs@SiO, alone }\end{array}$ \\
\hline $\begin{array}{l}\text { Au quantum } \\
\text { clusters }\end{array}$ & $\begin{array}{l}\text { Stabilizing ligand: } \\
\text { lipoic acid } \\
\text { Targeting ligand: FA } \\
\text { Photosensitizer: PPIX }\end{array}$ & Glioma (C6 cells) & $\begin{array}{l}\text { S.C. xenograft } \\
\text { mouse }\end{array}$ & $\begin{array}{l}532 \mathrm{~nm} \text { laser } \\
1.5 \mathrm{~W} / \mathrm{cm}^{2} \\
15 \mathrm{~min}\end{array}$ & $\begin{array}{l}\text { Under the laser stimulation, singlet } \\
\text { oxygen efficiency of the resultant NPs was } \\
\text { significantly higher when compared with } \\
\text { that of the PPIX alone }\end{array}$ \\
\hline
\end{tabular}

Photosensitizer: PPIX

Abbreviations: ALG, alginate; AuNPs, gold NPs; C. difficile, Clostridium difficile; Ce6, chlorin e6; CHI, chitosan; DOX, doxorubicin; FA, folic acid; GNRs, gold nanorods; HA, hyaluronic acid; HPPH, 3-devinyl-3-(I'-hexyloxyethyl)pyropheophorbide; ICG, indocyanine green; iPS, Induced pluripotent stem; i.v., intravenous; LED, light-emitting diode; MPCMs, macrophage cell membranes; MSCs, mesenchymal stem cells; NIR, near-infrared; NPs, nanoparticles; PDT, photodynamic therapy; PEG, polyethylene glycol; PEI, polyethylenimine; PEI-PAsp (DIP/MEA), polyethylenimine-b-poly(2-diisopropylamino/2-mercaptoethylamine) ethyl aspartate; PhA, pheophorbide a; PLGA, poly(lactic-coglycolic acid); PPIX, protoporphyrin IX; PTT, photothermal therapy; PVP, polyvinylpyrrolidone; RB, Rose Bengal; RBC, red blood cell; ROS, reactive oxygen species; S.C., subcutaneous; SERS, surface-enhanced Raman spectroscopy; SPR, surface plasmon resonance.

of NPs with a hydrodynamic size of $<6 \mathrm{~nm}$, while those with particle sizes of $>8 \mathrm{~nm}$ are able to avoid kidney removal. ${ }^{175}$ While NPs with $>10 \mathrm{~nm}$ diameter may reduce renal filtration, larger particles (ie, $>100 \mathrm{~nm}$ ) can evoke the reticuloendothelial system (RES). ${ }^{176}$ The RES, also known as the mononuclear phagocyte system (MPS), is part of the immune system, which primarily involves the liver, spleen, and lymph nodes. ${ }^{176,177}$ Foreign materials will be removed 


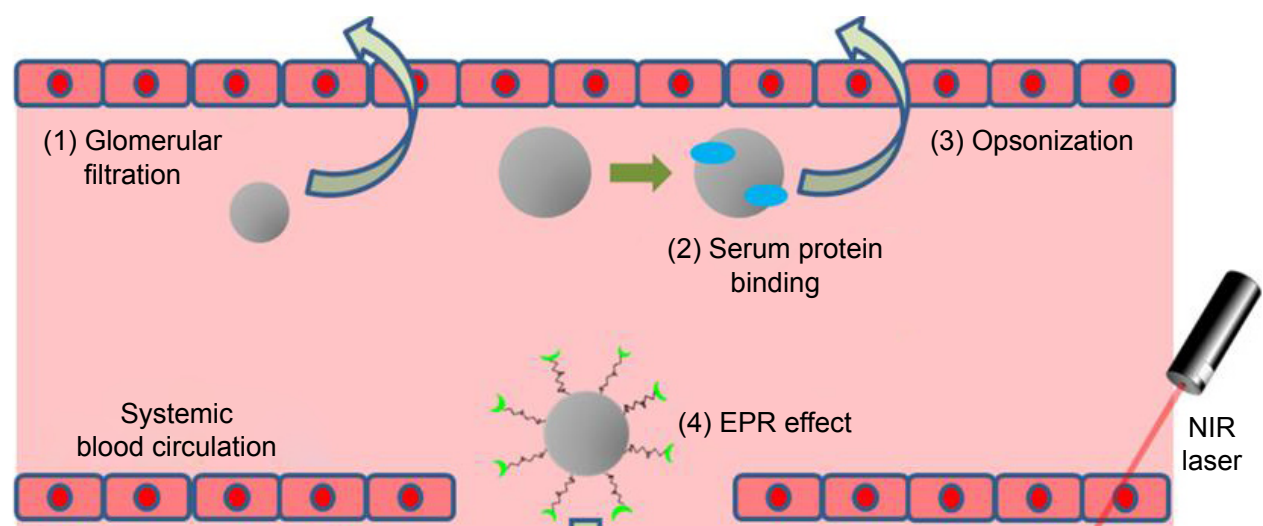

(5) Theranostics



Tumour imaging

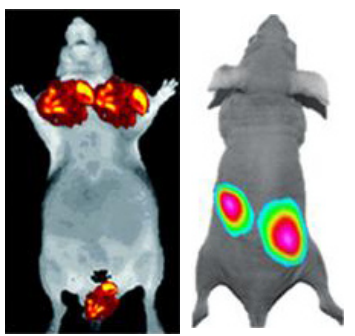

PTT

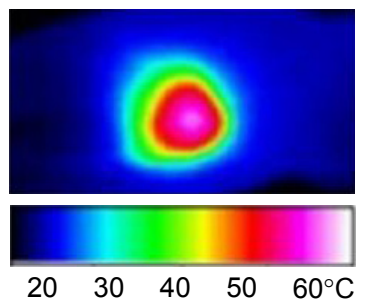

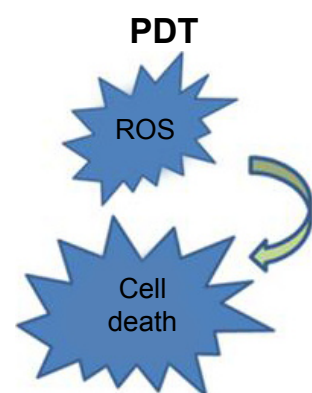

Figure 2 Systemic delivery of multifunctional AuNPs for cancer bioimaging and phototherapeutics.

Notes: (I) Following intravenous injection into the blood, AuNPs with a particle size of $<6 \mathrm{~nm}$ are prone to glomerular filtration. (2 and 3 ) When blood proteins bind to AuNPs nonspecifically, the resultant complexes tend to be taken up by MPS for opsonization (a means of identifying the invading particle to the phagocyte). (4) Multifunctional AuNPs $(\sim 100 \mathrm{~nm})$ with stealth coating materials, bioresponsive moieties, bioactive targeting ligands, and/or bioimaging agents can efficiently accumulate inside tumor tissues via the "EPR" effect and specifically target cancer cells via ligand-receptor pathway. (5) As a result, theranostic AuNPs can sensitively image the tumors and effectively induce PTT and/or PDT under the irradiation of the NIR light.

Abbreviations: AuNPs, gold nanoparticles; EPR, enhanced penetration and retention; MPS, mononuclear phagocyte system; NIR, near-infrared; PDT, photodynamic therapy; PTT, photothermal therapy; ROS, reactive oxygen species.

out of the blood circulation by the immune cells in MPS, thus dramatically reducing the circulatory half-time $\left(\mathrm{t}_{1 / 2}\right)$ of NPs. ${ }^{177}$ In addition, nonspecific adsorption of blood proteins onto the surface of NPs (ie, hydrophobic interactions between hydrophobic patches on proteins and hydrophobic ligands on NPs, and electrostatic interactions between anionic albumin and cationic NPs) may inhibit NPs' targeting capabilities or increase the overall particle size of NPs, and eventually the resultant large complexes are either trapped in the capillary endothelium of the lung or taken up by MPS. ${ }^{176}$

The modification of stabilizing ligands (ie, PEG, block copolymers, and hyaluronic acid [HA]) onto the gold surface, forming the so-called "stealth" particles capable of improving pharmacokinetic profiles, has been substantially reviewed previously. ${ }^{76,178,179}$ In addition to these stabilizing components, long-circulating NPs have also been developed where the NP surface is coated with the cellular membrane of red blood cells (RBCs) and leukocytes. ${ }^{150,154,174,175}$ As an alternative stealth coating material, the membrane of these cells completely covers the NP surface with their "selfmarkers" (ie, proteins, glycans, and acidic sialyl moieties), and the resultant NPs, recognized as the host's own cells, can actively evade the immune system. For example, Piao et al ${ }^{160}$ reported that the RBC-coated gold nanocages exhibited longer blood retention compared with the PEGylated counterparts. In addition, the fusion of RBC membranes over gold surface did not alter the unique porous and hollow structures of gold nanocages. Following systemic administration, the 
RBC-coated gold nanocages demonstrated enhanced tumor accumulation, induced NIR-active PTT, and eventually achieved $100 \%$ survival of tumor-bearing mice over a span of 45 days. ${ }^{160}$

\section{Targeting to specific cells of interest}

It is known that tumor grows rapidly via a process termed angiogenesis (new blood vessels are developed from the preexisting vasculature). ${ }^{177,180}$ The porous vasculature of a tumor is known to provide access to blood-circulating particles with sizes $<500 \mathrm{~nm}$, usually $<150 \mathrm{~nm} .{ }^{176}$ In addition, the lymphatic drainage system of tumor tissues is normally underdeveloped, and thus, NPs may not be removed. This EPR effect will facilitate "passive" accumulation of NPs in tumor tissues. In addition, targeting ligands (ie, antibodies and peptides) can be functionalized on NPs for "active" cancer targeting. Taking advantage of the "passive" and "active" targeting, recent advances in the design of AuNPs have offered great potential for accurate diagnosis and targeted therapy. For example, targeted gold nanocages were developed by coating with HA (a targeting ligand for CD44) on the gold surface to specifically recognize cancer cells overexpressing CD44. ${ }^{159}$ The HA-Au nanocages containing DOX could be efficiently uptaken by a receptor-mediated process, and subsequently, the coated HA molecules were degraded in lysosomes, resulting in the release of DOX. Biodistribution results demonstrate that targeted gold nanocages achieved significantly higher tumor accumulation relative to nontargeted counterparts in tumor-bearing mice. As a result, HAcoated gold nanocages containing DOX significantly slowed down the tumor growth, and more importantly, complete tumor inhibition was achieved when combined with PTT under the NIR stimulation. ${ }^{159}$

\section{Activation in response to TME}

TME is specifically adapted to support the growth, invasion, and metastasis of primary tumor tissues; this has been substantially reviewed previously. ${ }^{181}$ "Smart" bioresponsive NPs have been developed for targeted delivery and controlled drug release by exploiting the TME, where a slightly acidic environment, a low oxygen (hypoxia) niche, and overexpression of extracellular matrix (ECM) components (ie, MMPs) are evident. ${ }^{181}$

It is now known that the hypoxia region in solid tumors is a complex microenvironment with a low oxygen concentration and deficient nutrients. ${ }^{182}$ The hypoxic environment can not only lower the susceptibility of cancer cells to anticancer drugs but also reduce the response of cancer cells to free radicals. ${ }^{182}$
Although the hypoxia of solid tumors causes a hurdle in therapy, the low level of oxygen microenvironment serves as an ideal habitat for a number of anaerobic bacteria. Recently, Luo et al $^{149}$ reported a tumor-targeted AuNP delivery using two anaerobic bacterial strains, namely, Bifidobacterium breve UCC2003 and Clostridium difficile CCUG 37780. In this study, two approaches were designed for the active NP delivery: 1) a direct conjugation of GNRs on the surface of the vegetative $B$. breve for intravenous delivery into hypoxic region (a cargo-carrying approach) and 2) the injection of the $C$. difficile spores first, followed by the intravenous administration of the antibody-GNR conjugates to specifically target the germination of the $C$. difficile spores (an antibody-guiding approach). Under NIR excitation, the antibody-directed strategy showed stronger imaging and achieved effective PTT to completely inhibit tumor growth in a subcutaneous mouse cancer model. ${ }^{149}$

In addition, Sun et $\mathrm{al}^{93}$ developed novel AuNPs for dual CT/optical imaging of cancer. First, AuNPs were modified with glycol chitosan (GC) polymers (GC-AuNPs) for excellent stability and enhanced EPR effect. Second, fluorescent probes were chemically modified to GC-AuNPs via MMPactive peptides (MMP-GC-AuNPs). The NIR fluorescent probes were strongly quenched by the combinational quenching effects between the organic black hole quencher and the gold surface, but the quenched probes were reactivated upon exposure to MPPs in the TME. As a result, CT images with high spatial resolution and optical images with high sensitivity were simultaneously achieved using these AuNP-based CT/optical dual imaging probes. ${ }^{93}$

\section{Biodistribution, metabolism, and nanotoxicity}

In addition to tumor accumulation via the EPR effect, a majority of intravenously administrated NPs are nonspecifically distributed into healthy tissues (ie, phagocytic cell-rich organs such as the liver and spleen) before the renal clearance. ${ }^{183}$ Although the gold core is generally inert, nontoxic, and biocompatible, significant toxicity of AuNPs can be induced by the synthesis method of gold nanostructures, physicochemical properties (ie, size, morphology, and surface charge), surface conjugates and ligands, the doses, and the administration routes. ${ }^{173}$ Recently, numerous studies have described AuNPs designed to investigate whether their in vivo behaviors such as accumulation in healthy tissues and renal clearance cause unwanted effects. ${ }^{184-187}$

For example, the glomerular filtration, biodistribution, and toxicity of glutathione (GSH)- and bovine serum albumin 
(BSA)-capped AuNPs were evaluated using mice. ${ }^{188}$ As a result, smaller AuNPs (GSH-coated, 2 nm) caused highly efficient kidney removal and were more readily metabolized relative to the larger AuNPs (BSA-coated, 8 nm), suggesting that the toxicity was related to the size of AuNPs. In addition, although both AuNPs caused acute infection, inflammation, and kidney function damage after $24 \mathrm{~h}$, these effects were eliminated for $\sim 2 \mathrm{~nm}$ AuNPs after 28 days; in contrast, $~ 8 \mathrm{~nm}$ AuNPs further accumulated in the liver and spleen causing irreparable toxicity. ${ }^{188}$

It has been difficult to substantially evaluate the biodistribution, clearance, and toxicity of AuNPs, due to the fact that experimental designs are diverse, including the particle size, charge and shape, functionalization methods, types of animal models, delivery doses, and administration routes. Therefore, standard approaches are urgently needed in the future to facilitate development and approval of AuNPs for cancer theranostics. ${ }^{189}$

\section{Conclusion and future perspectives}

The newly emerging concept termed theranostics is well established as the development of novel strategies to combine diagnostic and therapeutic capabilities into a single agent facilitating specific and personalized therapies for diseases. ${ }^{190}$ The rationale behind the concept is that diseases, such as cancers, are extremely heterogeneous, and all existing treatments are effective for only certain patients and at selective stages of disease development. ${ }^{191}$ Therefore, a combined approach of diagnostics and therapeutics may provide promising treatment protocols that are more specific to individuals, ie, personalized medicines, and therefore more likely to offer improved prognoses.

Recent advances in understanding the optical and chemical properties of gold have enabled the design of novel AuNPs containing diagnostic and therapeutic functions that can be integrated into one system. ${ }^{40,42,192} \mathrm{Au}$-nanocomplex-based cancer imaging and phototherapy are reviewed in the "AuNPs for cancer diagnosis" and "AuNPs for cancer phototherapy" sections, respectively (Tables 1 and 2); many of these Au nanocomplexes, by tuning the size, shape, and structure, and by using different functional ligands, are capable of improving sensitive cancer diagnosis and targeted phototherapy simultaneously, under the stimulation of the appropriate light resources (NIR light is preferred in most cases). In addition, a number of AuNP-based theranostics have advanced into clinical trials (NCT03020017, NCT01270139 NCT02755870, NCT01420588, and NCT02782026). For example, the safety evaluation of a drug named NU-0129 is now undertaking an early Phase I trial (NCT03020017). NU-0129 is composed of
siRNA arranged on the surface of Au nanospheres, and when infused in patients with recurrent glioblastoma multiforme or gliosarcoma, the Au nanocomplexes can penetrate the bloodbrain barrier and deliver siRNA into tumor cells knocking down the expression of oncoprotein Bcl2Like12, which may result in significant inhibition of tumor growth (NCT03020017). Furthermore, CNM-Au8 (a drug candidate now being tested in clinical trial), an Au nanocrystal suspension drug, has recently been developed by Clene Nanomedicine (Salt Lake City, UT, USA) using Clean-Surface Nanosuspension ${ }^{\mathrm{TM}}$ (CSN) technology (it produces atomically clean-surface elemental nanocrystals, free of any residual surface chemicals, or surfacecapping agents) (www.clene.com). The oral administration of CNM-Au8 has demonstrated efficient immunomodulation in established demyelination animal models (www.clene.com). The safety, tolerability, and pharmacokinetics of CNM-Au8 are now under evaluation in healthy volunteers, with a hope of facilitating the application of the drug for the demyelinating disorder neuromyelitis optica (NMO) in the future (NCT02755870).

To overcome in vivo delivery barriers, Au nanocomplexes are normally modified with functional moieties such as stabilizing materials, targeting ligands, and bioresponsive linkers. However, it should be borne in mind that extensive functional modifications may cause unwanted toxic side effects, and therefore, further investigation is required to quantify the benefit of a treatment versus the risk of toxicity before theranostic Au nanocomplexes can be translated into clinically accepted strategies for cancer therapy.

\section{Acknowledgments}

We acknowledge the funding provided by the Outstanding Youth Foundation from the Department of Science and Technology, Jilin Province (Project Number: 20170520046JH); the Start-Up Research Grant Program from Jilin University (Project Number: 451160102052); the HAI-Novartis Fellowship Award from the Haematology Association of Ireland and Novartis Oncology; and the CNRS Lebanon GRP2015.

\section{Disclosure}

The authors report no conflicts of interest in this work.

\section{References}

1. Lee DE, Koo H, Sun IC, Ryu JH, Kim K, Kwon IC. Multifunctional nanoparticles for multimodal imaging and theragnosis. Chem Soc Rev. 2012; 41(7):2656-2672.

2. Ryu JH, Koo H, Sun IC, et al. Tumor-targeting multi-functional nanoparticles for theragnosis: new paradigm for cancer therapy. Adv Drug Deliv Rev. 2012;64(13):1447-1458.

3. Ahmed N, Fessi H, Elaissari A. Theranostic applications of nanoparticles in cancer. Drug Discov Today. 2012;17(17-18):928-934. 
4. Lim EK, Kim T, Paik S, Haam S, Huh YM, Lee K. Nanomaterials for theranostics: recent advances and future challenges. Chem Rev. 2015;115(1):327-394.

5. Namiki Y, Fuchigami T, Tada N, et al. Nanomedicine for cancer: lipidbased nanostructures for drug delivery and monitoring. Acc Chem Res. 2011;44(10):1080-1093.

6. Doane TL, Burda C. The unique role of nanoparticles in nanomedicine: imaging, drug delivery and therapy. Chem Soc Rev. 2012;41(7): 2885-2911.

7. Faraday M. The Bakerian Lecture: experimental relations of gold (and other metals) to light. Philos T R Soc A. 1857;147:145-181.

8. Turkevich J, Stevenson PC, Hillier J. A study of the nucleation and growth processes in the synthesis of colloidal gold. Discuss Faraday Soc. 1951;11:55-75.

9. Frens G. Controlled nucleation for the regulation of the particle size in monodisperse gold suspensions. Nature. 1973;241(105): 20-22.

10. Khoury CG, Vo-Dinh T. Gold nanostars for surface-enhanced Raman scattering: synthesis, characterization and optimization. J Phys Chem C Nanomater Interfaces. 2008;2008(112):18849-18859.

11. Dreaden EC, Alkilany AM, Huang X, Murphy CJ, El-Sayed MA. The golden age: gold nanoparticles for biomedicine. Chem Soc Rev. 2012; 41(7):2740-2779.

12. Vigderman L, Zubarev ER. Therapeutic platforms based on gold nanoparticles and their covalent conjugates with drug molecules. Adv Drug Deliv Rev. 2013;65(5):663-676.

13. Singhana B, Slattery P, Chen A, Wallace M, Melancon MP. Lightactivatable gold nanoshells for drug delivery applications. AAPS PharmSciTech. 2014;15(3):741-752.

14. Chen W, Zhang S, Yu Y, Zhang H, He Q. Structural-engineering rationales of gold nanoparticles for cancer theranostics. Adv Mater Deerfield. 2016;28(39):8567-8585.

15. Brown KR, Lyon LA, Fox AP, Reiss BD, Natan MJ. Hydroxylamine seeding of colloidal Au nanoparticles. 3. Controlled formation of conductive Au films. Chem Mater. 2000;12(2):314-323.

16. Rahme K, Nolan MT, Doody T, et al. Highly stable PEGylated gold nanoparticles in water: applications in biology and catalysis. RSC Adv. 2013;3(43):21016-21024.

17. Sau TK, Pal A, Jana NR, Wang ZL, Pal T. Size controlled synthesis of gold nanoparticles using photochemically prepared seed particles. J Nanopart Res. 2001;3(4):257-261.

18. Niu JL, Zhu T, Liu ZF. One-step seed-mediated growth of 30-150 nm quasispherical gold nanoparticles with 2-mercaptosuccinic acid as a new reducing agent. Nanotechnology. 2007;18(32):7.

19. Perrault SD, Chan WCW. Synthesis and surface modification of highly monodispersed, spherical gold nanoparticles of 50-200 nm. J Am Chem Soc. 2009;131(47):17042.

20. Liu X, Xu H, Xia H, Wang D. Rapid seeded growth of monodisperse, quasi-spherical, citrate-stabilized gold nanoparticles via $\mathrm{H}_{2} \mathrm{O}_{2}$ reduction. Langmuir. 2012;28(38):13720-13726.

21. Foss CA, Hornyak GL, Stockert JA, Martin CR. Optical-properties of composite membranes containing arrays of nanoscopic gold cylinders. J Phys Chem. 1992;96(19):7497-7499.

22. Martin CR. Nanomaterials: a membrane-based synthetic approach. Science. 1994;266(5193):1961-1966.

23. Perez-Juste J, Pastoriza-Santos I, Liz-Marzan LM, Mulvaney P. Gold nanorods: synthesis, characterization and applications. Coordin Chem Rev. 2005;249(17-18):1870-1901.

24. Jana NR, Gearheart L, Murphy CJ. Evidence for seed-mediated nucleation in the chemical reduction of gold salts to gold nanoparticles. Chem Mater. 2001;13(7):2313-2322.

25. Nikoobakht B, El-Sayed MA. Preparation and growth mechanism of gold nanorods (NRs) using seed-mediated growth method. Chem Mater. 2003;15(10):1957-1962.

26. Skrabalak SE, Chen JY, Sun YG, et al. Gold nanocages: synthesis, properties, and applications. Acc Chem Res. 2008;41(12): 1587-1595.
27. Yong KT, Sahoo Y, Swihart MT, Prasad PN. Synthesis and plasmonic properties of silver and gold nanoshells on polystyrene cores of different size and of gold-silver core-shell nanostructures. Colloid Surf A. 2006;290(1-3):89-105.

28. Oldenburg SJ, Averitt RD, Westcott SL, Halas NJ. Nanoengineering of optical resonances. Chem Phys Lett. 1998;288(2-4):243-247.

29. Pham T, Jackson JB, Halas NJ, Lee TR. Preparation and characterization of gold nanoshells coated with self-assembled monolayers. Langmuir. 2002;18(12):4915-4920.

30. Prodan E, Radloff C, Halas NJ, Nordlander P. A hybridization model for the plasmon response of complex nanostructures. Science. 2003; 302(5644):419-422.

31. Kreibig U, Vollmer M. Optical Properties of Metal Clusters. Berlin Heidelberg: Springer-Verlag; 1995.

32. Cobley CM, Chen JY, Cho EC, Wang LV, Xia YN. Gold nanostructures: a class of multifunctional materials for biomedical applications. Chem Soc Rev. 2011;40(1):44-56.

33. Huang X, El-Sayed MA. Gold nanoparticles: optical properties and implementations in cancer diagnosis and photothermal therapy. $J A d v$ Res. 2010;1:13-28.

34. Jain PK, Lee KS, El-Sayed IH, El-Sayed MA. Calculated absorption and scattering properties of gold nanoparticles of different size, shape, and composition: applications in biological imaging and biomedicine. J Phys Chem B. 2006;110(14):7238-7248.

35. Yguerabide J, Yguerabide EE. Light-scattering submicroscopic particles as highly fluorescent analogs and their use as tracer labels in clinical and biological applications - I. Theory. Anal Biochem. 1998;262(2): 137-156.

36. Yguerabide J, Yguerabide EE. Light-scattering submicroscopic particles as highly fluorescent analogs and their use as tracer labels in clinical and biological applications - II. Experimental characterization. Anal Biochem. 1998;262(2):157-176.

37. Sokolov K, Follen M, Aaron J, et al. Real-time vital optical imaging of precancer using anti-epidermal growth factor receptor antibodies conjugated to gold nanoparticles. Cancer Res. 2003;63(9):1999-2004.

38. Orendorff CJ, Sau TK, Murphy CJ. Shape-dependent plasmon-resonant gold nanoparticles. Small. 2006;2(5):636-639.

39. Lee KS, El-Sayed MA. Dependence of the enhanced optical scattering efficiency relative to that of absorption for gold metal nanorods on aspect ratio, size, end-cap shape, and medium refractive index. $J$ Phys Chem B. 2005;109(43):20331-20338

40. Hauck TS, Chan WC. Gold nanoshells in cancer imaging and therapy: towards clinical application. Nanomedicine (Lond). 2007;2(5): 735-738.

41. Skrabalak SE, Au L, Lu X, Li X, Xia Y. Gold nanocages for cancer detection and treatment. Nanomedicine (Lond). 2007;2(5):657-668.

42. Huang X, Neretina S, El-Sayed MA. Gold nanorods: from synthesis and properties to biological and biomedical applications. Adv Mater. 2009;21(48):4880-4910.

43. Tong L, Wei QS, Wei A, Cheng JX. Gold nanorods as contrast agents for biological imaging: optical properties, surface conjugation and photothermal effects. Photochem Photobiol. 2009;85(1):21-32.

44. Lee KS, El-Sayed MA. Gold and silver nanoparticles in sensing and imaging: sensitivity of plasmon response to size, shape, and metal composition. J Phys Chem B. 2006;110(39):19220-19225.

45. Chen J, Saeki F, Wiley BJ, et al. Gold nanocages: bioconjugation and their potential use as optical imaging contrast agents. Nano Lett. 2005; $5(3): 473-477$.

46. Sun YG, Mayers BT, Xia YN. Template-engaged replacement reaction: a one-step approach to the large-scale synthesis of metal nanostructures with hollow interiors. Nano Lett. 2002;2(5):481-485.

47. Agasti SS, Rana S, Park MH, Kim CK, You CC, Rotello VM. Nanoparticles for detection and diagnosis. Adv Drug Deliv Rev. 2010;62(3): 316-328.

48. Portales H, Saviot L, Duval E, et al. Resonance and composition effects on the Raman scattering from silver-gold alloy clusters. Eur Phys J D. 2001;16(1-3):197-200. 
49. Amendola V, Scaramuzza S, Agnoli S, Polizzi S, Meneghetti M. Strong dependence of surface plasmon resonance and surface enhanced Raman scattering on the composition of Au-Fe nanoalloys. Nanoscale. 2014;6(3):1423-1433.

50. Juluri BK, Zheng YB, Ahmed D, Jensen L, Huang TJ. Effects of geometry and composition on charge-induced plasmonic shifts in gold nanoparticles. J Phys Chem C. 2008;112(19):7309-7317.

51. Brust M, Walker M, Bethell D, Schiffrin DJ, Whyman R. Synthesis of thiol-derivatized gold nanoparticles in a 2-phase liquid-liquid system. J Chem Soc Chem Comm. 1994;(7):801-802.

52. Sardar R, Funston AM, Mulvaney P, Murray RW. Gold nanoparticles: past, present, and future. Langmuir. 2009;25(24):13840-13851.

53. Guo J, Armstrong MJ, O’Driscoll CM, Holmes JD, Rahme K. Positively charged, surfactant-free gold nanoparticles for nucleic acid delivery. RSC Adv. 2015;5:17862-17871.

54. Chhour P, Naha PC, O’Neill SM, et al. Labeling monocytes with gold nanoparticles to track their recruitment in atherosclerosis with computed tomography. Biomaterials. 2016;87:93-103.

55. Liu XS, Huang N, Wang HB, Li H, Jin Q, Ji J. The effect of ligand composition on the in vivo fate of multidentate poly(ethylene glycol) modified gold nanoparticles. Biomaterials. 2013;34(33):8370-8381.

56. Conde J, Tian F, Hernandez Y, et al. In vivo tumor targeting via nanoparticle-mediated therapeutic siRNA coupled to inflammatory response in lung cancer mouse models. Biomaterials. 2013;34(31):7744-7753.

57. Shiao YS, Chiu HH, Wu PH, Huang YF. Aptamer-functionalized gold nanoparticles as photoresponsive nanoplatform for co-drug delivery. ACS Appl Mater Interfaces. 2014;6(24):21832-21841.

58. Arosio D, Chiodo F, Reina JJ, et al. Effective targeting of DC-SIGN by alpha-fucosylamide functionalized gold nanoparticles. Bioconjugate Chem. 2014;25(12):2244-2251.

59. Fitzgerald KA, Rahme K, Guo JF, Holmes JD, O’Driscoll CM. Anisamide-targeted gold nanoparticles for siRNA delivery in prostate cancer - synthesis, physicochemical characterisation and in vitro evaluation. J Mater Chem B. 2016;4(13):2242-2252.

60. Guo J, O’Driscoll CM, Holmes JD, Rahme K. Bioconjugated gold nanoparticles enhance cellular uptake: a proof of concept study for siRNA delivery in prostate cancer cells. Int J Pharm. 2016;509(1-2):16-27.

61. Shen S, Tang HY, Zhang XT, et al. Targeting mesoporous silicaencapsulated gold nanorods for chemo-photothermal therapy with near-infrared radiation. Biomaterials. 2013;34(12):3150-3158.

62. Guo JF, Ogier JR, Desgranges S, Darcy R, O’Driscoll C. Anisamidetargeted cyclodextrin nanoparticles for siRNA delivery to prostate tumours in mice. Biomaterials. 2012;33(31):7775-7784.

63. Fitzgerald KA, Malhotra M, Gooding M, et al. A novel, anisamidetargeted cyclodextrin nanoformulation for siRNA delivery to prostate cancer cells expressing the sigma-1 receptor. Int J Pharm. 2016; 499(1-2):131-145.

64. Stolik S, Delgado JA, Perez A, Anasagasti L. Measurement of the penetration depths of red and near infrared light in human "ex vivo" tissues. J Photochem Photobiol B. 2000;57(2-3):90-93.

65. Sakudo A. Near-infrared spectroscopy for medical applications: current status and future perspectives. Clin Chim Acta. 2016;455:181-188.

66. Henderson TA, Morries LD. Near-infrared photonic energy penetration: can infrared phototherapy effectively reach the human brain? Neuropsych Dis Treat. 2015;11:2191-2208.

67. El-Sayed IH, Huang X, El-Sayed MA. Surface plasmon resonance scattering and absorption of anti-EGFR antibody conjugated gold nanoparticles in cancer diagnostics: applications in oral cancer. Nano Lett. 2005;5(5):829-834.

68. Choi J, Yang J, Bang D, et al. Targetable gold nanorods for epithelial cancer therapy guided by near-IR absorption imaging. Small. 2012; 8(5):746-753.

69. Yang XM, Stein EW, Ashkenazi S, Wang LHV. Nanoparticles for photoacoustic imaging. Wires Nanomed Nanobi. 2009;1(4): 360-368.

70. Wang H, Brandl DW, Nordlander P, Halas NJ. Plasmonic nanostructures: artificial molecules. Acc Chem Res. 2007;40(1):53-62.
71. Halas NJ, Lal S, Chang WS, Link S, Nordlander P. Plasmons in strongly coupled metallic nanostructures. Chem Rev. 2011;111(6):3913-3961.

72. Li W, Chen X. Gold nanoparticles for photoacoustic imaging. Nanomedicine (Lond). 2015;10(2):299-320.

73. Song JB, Yang XY, Jacobson O, et al. Ultrasmall gold nanorod vesicles with enhanced tumor accumulation and fast excretion from the body for cancer therapy. Adv Mater Deerfield. 2015;27(33):4910-4917.

74. Popovtzer R, Agrawal A, Kotov NA, et al. Targeted gold nanoparticles enable molecular CT imaging of cancer. Nano Lett. 2008;8(12): 4593-4596.

75. Liu H, Shen M, Zhao J, et al. Tunable synthesis and acetylation of dendrimer-entrapped or dendrimer-stabilized gold-silver alloy nanoparticles. Colloids Surf B Biointerfaces. 2012;94:58-67.

76. Jokerst JV, Lobovkina T, Zare RN, Gambhir SS. Nanoparticle PEGylation for imaging and therapy. Nanomedicine (Lond). 2011;6(4): 715-728.

77. Zhou B, Yang J, Peng C, et al. PEGylated polyethylenimine-entrapped gold nanoparticles modified with folic acid for targeted tumor CT imaging. Colloids Surf B Biointerfaces. 2016;140:489-496.

78. Paul AM, Fan Z, Sinha SS, et al. Bioconjugated gold nanoparticle based SERS probe for ultrasensitive identification of mosquitoborne viruses using Raman fingerprinting. J Phys Chem C. 2015; 119(41):23669-23675.

79. Huang X, El-Sayed IH, Qian W, El-Sayed MA. Cancer cells assemble and align gold nanorods conjugated to antibodies to produce highly enhanced, sharp, and polarized surface Raman spectra: a potential cancer diagnostic marker. Nano Lett. 2007;7(6):1591-1597.

80. Hoejgaard L, Hesse B. Hybrid imaging: conclusions and perspectives. Curr Med Imaging Rev. 2011;7(3):252-253.

81. Meir R, Motiei M, Popovtzer R. Gold nanoparticles for in vivo cell tracking. Nanomedicine (Lond). 2014;9(13):2059-2069.

82. Jin Y. Multifunctional compact hybrid Au nanoshells: a new generation of nanoplasmonic probes for biosensing, imaging, and controlled release. Acc Chem Res. 2014;47(1):138-148.

83. Zhao J, Wallace M, Melancon MP. Cancer theranostics with gold nanoshells. Nanomedicine. 2014;9(13):2041-2057.

84. Zhao Y, Pang B, Luehmann H, et al. Gold nanoparticles doped with (199) Au atoms and their use for targeted cancer imaging by SPECT. Adv Healthc Mater. 2016;5(8):928-935.

85. He XX, Liu FY, Liu L, Duan TC, Zhang HM, Wanq ZX. LectinconjugatedFe2O3@Au core@shell nanoparticles as dual mode contrast agents for in vivo detection of tumor. Mol Pharm. 2014;11(3): $738-745$.

86. Huang S, Li C, Wang WP, et al. A54 peptide-mediated functionalized gold nanocages for targeted delivery of DOX as a combinational photothermal-chemotherapy for liver cancer. Int $J$ Nanomedicine. 2017;12:5163-5176.

87. Zhang JM, Li C, Zhang X, et al. In vivo tumor-targeted dual-modal fluorescence/CT imaging using a nanoprobe co-loaded with an aggregation-induced emission dye and gold nanoparticles. Biomaterials. 2015;42:103-111.

88. Meir R, Shamalov K, Betzer O, et al. Nanomedicine for cancer immunotherapy: tracking cancer-specific T-cells in vivo with gold nanoparticles and CT imaging. ACS Nano. 2015;9(6):6363-6372.

89. Peng C, Zheng L, Chen Q, et al. PEGylated dendrimer-entrapped gold nanoparticles for in vivo blood pool and tumor imaging by computed tomography. Biomaterials. 2012;33(4):1107-1119.

90. Cheng B, He HC, Huang T, et al. Gold nanosphere gated mesoporous silica nanoparticle responsive to near-infrared light and redox potential as a theranostic platform for cancer therapy. J Biomed Nanotechnol. 2016;12(3):435-449.

91. Cheng Y, Dai Q, Morshed RA, et al. Blood-brain barrier permeable gold nanoparticles: an efficient delivery platform for enhanced malignant glioma therapy and imaging. Small. 2014;10(24):5137-5150.

92. Kim YH, Jeon J, Hong SH, et al. Tumor targeting and imaging using cyclic RGD-PEGylated gold nanoparticle probes with directly conjugated iodine-125. Small. 2011;7(14):2052-2060. 
93. Sun IC, Eun DK, Koo H, et al. Tumor-targeting gold particles for dual computed tomography/optical cancer imaging. Angew Chem Int Ed Engl. 2011;50(40):9348-9351.

94. Chen Q, Li KA, Wen SH, et al. Targeted CT/MR dual mode imaging of tumors using multifunctional dendrimer-entrapped gold nanoparticles. Biomaterials. 2013;34(21):5200-5209.

95. Jing LJ, Liang XL, Deng ZJ, et al. Prussian blue coated gold nanoparticles for simultaneous photoacoustic/CT bimodal imaging and photothermal ablation of cancer. Biomaterials. 2014;35(22): 5814-5821.

96. Gao S, Zhang LW, Wang GH, et al. Hybrid graphene/Au activatable theranostic agent for multimodalities imaging guided enhanced photothermal therapy. Biomaterials. 2016;79:36-45.

97. Chen WH, Yang CX, Qiu WX, et al. Multifunctional theranostic nanoplatform for cancer combined therapy based on gold nanorods. Adv Healthc Mater. 2015;4(15):2247-2259.

98. Jang B, Park JY, Tung CH, Kim IH, Choi Y. Gold nanorodphotosensitizer complex for near-infrared fluorescence imaging and photodynamic/photothermal therapy in vivo. ACS Nano. 2011;5(2): 1086-1094.

99. Yang HW, Liu HL, Li ML, et al. Magnetic gold-nanorod/PNIPAAmMA nanoparticles for dual magnetic resonance and photoacoustic imaging and targeted photothermal therapy. Biomaterials. 2013;34(22):5651-5660.

100. Jokerst JV, Cole AJ, Van de Sompel D, Gambhir SS. Gold nanorods for ovarian cancer detection with photoacoustic imaging and resection guidance via Raman imaging in living mice. ACS Nano. 2012;6(11):10366-10377.

101. Guan T, Shang W, Li H, et al. From detection to resection: photoacoustic tomography and surgery guidance with indocyanine green loaded gold nanorod@liposome core-shell nanoparticles in liver cancer. Bioconjug Chem. 2017;28(4):1221-1228.

102. Karmani L, Labar D, Valembois V, et al. Antibody-functionalized nanoparticles for imaging cancer: influence of conjugation to gold nanoparticles on the biodistribution of 897r-labeled cetuximab in mice. Contrast Media Mol Imaging. 2013;8(5):402-408.

103. Xie H, Wang ZJ, Bao A, Goins B, Phillips WT. In vivo PET imaging and biodistribution of radiolabeled gold nanoshells in rats with tumor xenografts. Int J Pharm. 2010;395(1-2):324-330.

104. Chen W, Ayala-Orozco C, Biswal NC, et al. Targeting pancreatic cancer with magneto-fluorescent theranostic gold nanoshells. Nanomedicine (Lond). 2014;9(8):1209-1222.

105. Coughlin AJ, Ananta JS, Deng NF, Larina IV, Decuzzi P, West JL. Gadolinium-conjugated gold nanoshells for multimodal diagnostic imaging and photothermal cancer therapy. Small. 2014;10(3):556-565.

106. Li L, Fu S, Chen C, et al. Microenvironment-driven bioelimination of magnetoplasmonic nanoassemblies and their multimodal imaging-guided tumor photothermal therapy. ACS Nano. 2016;10(7): 7094-7105.

107. Chen HY, Li BW, Ren XY, et al. Multifunctional near-infraredemitting nano-conjugates based on gold clusters for tumor imaging and therapy. Biomaterials. 2012;33(33):8461-8476.

108. Zhang P, Yang XX, Wang Y, Zhao NW, Xiong ZH, Huang CZ. Rapid synthesis of highly luminescent and stable Au-20 nanoclusters for active tumor-targeted imaging in vitro and in vivo. Nanoscale. 2014;6(4):2261-2269.

109. Croissant JG, Zhang D, Alsaiari S, et al. Protein-gold clusters-capped mesoporous silica nanoparticles for high drug loading, autonomous gemcitabine/doxorubicin co-delivery, and in-vivo tumor imaging. J Control Release. 2016;229:183-191.

110. Zhao YF, Sultan D, Detering L, Luehmann H, Liu YJ. Facile synthesis, pharmacokinetic and systemic clearance evaluation, and positron emission tomography cancer imaging of $\mathrm{Cu}-64-\mathrm{Au}$ alloy nanoclusters. Nanoscale. 2014;6(22):13501-13509.

111. Hu H, Huang P, Weiss OJ, et al. PET and NIR optical imaging using self-illuminating $\mathrm{Cu}$-64-doped chelator-free gold nanoclusters. Biomaterials. 2014;35(37):9868-9876.
112. Hu DH, Sheng ZH, Zhang PF, et al. Hybrid gold-gadolinium nanoclusters for tumor-targeted NIRF/CT/MRI triple-modal imaging in vivo. Nanoscale. 2013;5(4):1624-1628.

113. Han S, Samanta A, Xie X, et al. Gold and hairpin DNA functionalization of upconversion nanocrystals for imaging and in vivo drug delivery. Adv Mater Deerfield. Epub 2017 Mar 10;29(18).

114. Gao X, Yue Q, Liu Z, et al. Guiding brain-tumor surgery via bloodbrain-barrier-permeable gold nanoprobes with acid-triggered MRI/ SERRS signals. Adv Mater. Epub 2017 Mar 15;29(21).

115. Park J, Park J, Ju EJ, et al. Multifunctional hollow gold nanoparticles designed for triple combination therapy and CT imaging. J Control Release. 2015;207:77-85.

116. Tian M, Lu W, Zhang R, et al. Tumor uptake of hollow gold nanospheres after intravenous and intra-arterial injection: PET/CT study in a rabbit VX2 liver cancer model. Mol Imaging Biol. 2013; 15(5):614-624.

117. Lu W, Melancon MP, Xiong C, et al. Effects of photoacoustic imaging and photothermal ablation therapy mediated by targeted hollow gold nanospheres in an orthotopic mouse xenograft model of glioma. Cancer Res. 2011;71(19):6116-6121.

118. Liu Y, Ashton JR, Moding EJ, et al. A plasmonic gold nanostar theranostic probe for in vivo tumor imaging and photothermal therapy. Theranostics. 2015;5(9):946-960.

119. Huang J, Guo M, Ke HT, et al. Rational design and synthesis gamma Fe2O3@Au magnetic gold nanoflowers for efficient cancer theranostics. Adv Mater. 2015;27(34):5049.

120. Hou W, Xia F, Alfranca G, et al. Nanoparticles for multi-modality cancer diagnosis: simple protocol for self-assembly of gold nanoclusters mediated by gadolinium ions. Biomaterials. 2017;120: $103-114$.

121. D'hollander A, Mathieu E, Jans H, et al. Development of nanostars as a biocompatible tumor contrast agent: toward in vivo SERS imaging. Int J Nanomedicine. 2016;11:3703-3714.

122. Wang Y, Liu Y, Luehmann H, et al. Evaluating the pharmacokinetics and in vivo cancer targeting capability of Au nanocages by positron emission tomography imaging. ACS Nano. 2012;6(7):5880-5888.

123. Bao CC, Beziere N, del Pino P, et al. Gold nanoprisms as optoacoustic signal nanoamplifiers for in vivo bioimaging of gastrointestinal cancers. Small. 2013;9(1):68-74.

124. Cheng K, Kothapalli SR, Liu HG, et al. Construction and validation of nano gold tripods for molecular imaging of living subjects. $J \mathrm{Am}$ Chem Soc. 2014;136(9):3560-3571.

125. Link S, El-Sayed MA. Shape and size dependence of radiative, nonradiative and photothermal properties of gold nanocrystals. Int Rev Phys Chem. 2000;19(3):409-453.

126. Link S, Furube A, Mohamed MB, Asahi T, Masuhara H, El-Sayed MA. Hot electron relaxation dynamics of gold nanoparticles embedded in $\mathrm{MgSO}_{4}$ powder compared to solution: the effect of the surrounding medium. J Phys Chem B. 2002;106(5):945-955.

127. Link S, Ei-Sayed MA. Optical properties and ultrafast dynamics of metallic nanocrystals. Annu Rev Phys Chem. 2003;54:331-366.

128. Link S, Hathcock DJ, Nikoobakht B, El-Sayed MA. Medium effect on the electron cooling dynamics in gold nanorods and truncated tetrahedra. Adv Mater. 2003;15(5):393.

129. Riley RS, Day ES. Gold nanoparticle-mediated photothermal therapy: applications and opportunities for multimodal cancer treatment. Wiley interdisciplinary reviews. Nanomed Nanobiotechnol. 2017;9(4):e1449.

130. Huang XH, Jain PK, El-Sayed IH, El-Sayed MA. Gold nanoparticles: interesting optical properties and recent applications in cancer diagnostic and therapy. Nanomedicine. 2007;2(5):681-693.

131. Hwang S, Nam J, Jung S, Song J, Doh H, Kim S. Gold nanoparticlemediated photothermal therapy: current status and future perspective. Nanomedicine (Lond). 2014;9(13):2003-2022.

132. Huang X, Qian W, El-Sayed IH, El-Sayed MA. The potential use of the enhanced nonlinear properties of gold nanospheres in photothermal cancer therapy. Lasers Surg Med. 2007;39(9):747-753. 
133. El-Sayed IH, Huang XH, El-Sayed MA. Selective laser photo-thermal therapy of epithelial carcinoma using anti-EGFR antibody conjugated gold nanoparticles. Cancer Lett. 2006;239(1):129-135.

134. Hleb EY, Hafner JH, Myers JN, et al. LANTCET: elimination of solid tumor cells with photothermal bubbles generated around clusters of gold nanoparticles. Nanomedicine. 2008;3(5):647-667.

135. Li Z, Huang P, Zhang X, et al. RGD-conjugated dendrimer-modified gold nanorods for in vivo tumor targeting and photothermal therapy. Mol Pharm. 2010;7(1):94-104.

136. Ke HT, Wang JR, Tong S, et al. Gold nanoshelled liquid perfluorocarbon magnetic nanocapsules: a nanotheranostic platform for bimodal ultrasound/magnetic resonance imaging guided photothermal tumor ablation. Theranostics. 2014;4(1):12-23.

137. Ayala-Orozco C, Urban C, Bishnoi S, et al. Sub-100nm gold nanomatryoshkas improve photo-thermal therapy efficacy in large and highly aggressive triple negative breast tumors. J Control Release. 2014;191: 90-97.

138. Allison RR, Moghissi K. Photodynamic therapy (PDT): PDT mechanisms. Clin Endosc. 2013;46(1):24-29.

139. Schweitzer VG, Somers ML. PHOTOFRIN-mediated photodynamic therapy for treatment of early stage (Tis-T2N0M0) SqCCa of oral cavity and oropharynx. Laser Surg Med. 2010;42(1):1-8.

140. Vankayala R, Sagadevan A, Vijayaraghavan P, Kuo CL, Hwang KC. Metal nanoparticles sensitize the formation of singlet oxygen. Angew Chem Int Ed Engl. 2011;50(45):10640-10644.

141. Vankayala R, Lin CC, Kalluru P, Chiang CS, Hwang KC. Gold nanoshells-mediated bimodal photodynamic and photothermal cancer treatment using ultra-low doses of near infra-red light. Biomaterials. 2014;35(21):5527-5538.

142. Chen R, Wang X, Yao XK, Zheng XC, Wang J, Jiang XQ. NearIR-triggered photothermal/photodynamic dual-modality therapy system via chitosan hybrid nanospheres. Biomaterials. 2013;34(33): 8314-8322.

143. von Maltzahn G, Park JH, Agrawal A, et al. Computationally guided photothermal tumor therapy using long-circulating gold nanorod antennas. Cancer Res. 2009;69(9):3892-3900.

144. Sun XL, Huang XL, Yan XF, et al. Chelator-free Cu-64-integrated gold nanomaterials for positron emission tomography imaging guided photothermal cancer therapy. ACS Nano. 2014;8(8): 8438-8446.

145. Zhang Z, Wang J, Nie X, et al. Near infrared laser-induced targeted cancer therapy using thermoresponsive polymer encapsulated gold nanorods. J Am Chem Soc. 2014;136(20):7317-7326.

146. Li XJ, Takashima M, Yuba E, Harada A, Kono K. PEGylated PAMAM dendrimer-doxorubicin conjugate-hybridized gold nanorod for combined photothermal-chemotherapy. Biomaterials. 2014; 35(24):6576-6584.

147. Shi H, Ye XS, He XX, et al. Au@Ag/Au nanoparticles assembled with activatable aptamer probes as smart "nano-doctors" for image-guided cancer thermotherapy. Nanoscale. 2014;6(15):8754-8761.

148. Shao JX, Xuan MJ, Dai LR, Si TY, Li JB, He Q. Near-infraredactivated nanocalorifiers in microcapsules: vapor bubble generation for in vivo enhanced cancer therapy. Angew Chem Int Edit. 2015; 54(43): 12782-12787.

149. Luo CH, Huang CT, Su CH, Yeh CS. Bacteria-mediated hypoxiaspecific delivery of nanoparticles for tumors imaging and therapy. Nano Lett. 2016;16(6):3493-3499.

150. Wang $\mathrm{X}$, Wang $\mathrm{H}$, Wang $\mathrm{Y}$, et al. A facile strategy to prepare dendrimer-stabilized gold nanorods with sub-10-nm size for efficient photothermal cancer therapy. Sci Rep. 2016;6:22764.

151. Liu Y, Yang M, Zhang J, et al. Human induced pluripotent stem cells for tumor targeted delivery of gold nanorods and enhanced photothermal therapy. ACS Nano. 2016;10(2):2375-2385.

152. Ayala-Orozco C, Urban C, Knight MW, et al. Au nanomatryoshkas as efficient near-infrared photothermal transducers for cancer treatment: benchmarking against nanoshells. ACS Nano. 2014;8(6): 6372-6381.
153. Li W, Zhang XJ, Zhou MJ, et al. Functional core/shell drug nanoparticles for highly effective synergistic cancer therapy. Adv Healthc Mater. 2014;3(9):1475-1485.

154. Hao YW, Zhang BX, Zheng CX, et al. The tumor-targeting coreshell structured DTX-loaded PLGA@Au nanoparticles for chemophotothermal therapy and X-ray imaging. J Control Release. 2015; 220(pt A):545-555.

155. Wang L, Yuan YY, Lin SD, et al. Photothermo-chemotherapy of cancer employing drug leakage-free gold nanoshells. Biomaterials. 2016;78:40-49.

156. Xuan M, Shao J, Dai L, Li J, He Q. Macrophage cell membrane camouflaged Au nanoshells for in vivo prolonged circulation life and enhanced cancer photothermal therapy. ACS Appl Mater Interfaces. 2016;8(15):9610-9618.

157. Nie LM, Wang SJ, Wang XY, et al. In vivo volumetric photoacoustic molecular angiography and therapeutic monitoring with targeted plasmonic nanostars. Small. 2014;10(8):1585-1593.

158. Gao YP, Li YS, Chen JZ, et al. Multifunctional gold nanostarbased nanocomposite: synthesis and application for noninvasive MR-SERS imaging-guided photothermal ablation. Biomaterials. 2015;60:31-41

159. Wang ZZ, Chen ZW, Liu Z, et al. A multi-stimuli responsive gold nanocage-hyaluronic platform for targeted photothermal and chemotherapy. Biomaterials. 2014;35(36):9678-9688.

160. Piao JG, Wang LM, Gao F, You YZ, Xiong YJ, Yang LH. Erythrocyte membrane is an alternative coating to polyethylene glycol for prolonging the circulation lifetime of gold nanocages for photothermal therapy. ACS Nano. 2014;8(10):10414-10425.

161. Wang ZH, Sun JH, Qiu YQ, et al. Specific photothermal therapy to the tumors with high EphB4 receptor expression. Biomaterials. 2015 68:32-41.

162. Zhou JL, Wang ZH, Li QP, et al. Hybridized doxorubicin-Au nanospheres exhibit enhanced near-infrared surface plasmon absorption for photothermal therapy applications. Nanoscale. 2015;7(13): 5869-5883.

163. Kang S, Bhang SH, Hwang S, et al. Mesenchymal stem cells aggregate and deliver gold nanoparticles to tumors for photothermal therapy. ACS Nano. 2015;9(10):9678-9690.

164. Chen M, Tang SH, Guo ZD, et al. Core-shell Pd@Au nanoplates as theranostic agents for in-vivo photoacoustic imaging, CT imaging, and photothermal therapy. Adv Mater. 2014;26(48):8210-8216.

165. Li L, Nurunnabi M, Nafiujjaman M, Lee YK, Huh KM. GSHmediated photoactivity of pheophorbide a-conjugated heparin/gold nanoparticle for photodynamic therapy. J Control Release. 2013; 171(2):241-250.

166. Zhang Y, Qian J, Wang D, Wang YL, He SL. Multifunctional gold nanorods with ultrahigh stability and tunability for in vivo fluorescence imaging, SERS detection, and photodynamic therapy. Angew Chem Int Edit. 2013;52(4):1148-1151.

167. Kim JY, Choi WI, Kim M, Tae G. Tumor-targeting nanogel that can function independently for both photodynamic and photothermal therapy and its synergy from the procedure of PDT followed by PTT. J Control Release. 2013;171(2):113-121.

168. Vankayala R, Huang YK, Kalluru P, Chiang CS, Hwang KC. First demonstration of gold nanorods-mediated photodynamic therapeutic destruction of tumors via near infra-red light activation. Small. 2014; 10(8):1612-1622.

169. Wang BK, Wang JH, Liu Q, et al. Rose-bengal-conjugated gold nanorods for in vivo photodynamic and photothermal oral cancer therapies. Biomaterials. 2014;35(6):1954-1966.

170. Ye SF, Kang N, Chen M, et al. Tat/HA2 peptides conjugated AuNR@pNIPAAm as a photosensitizer carrier for near infrared triggered photodynamic therapy. Mol Pharm. 2015;12(7): 2444-2458.

171. Srivatsan A, Jenkins SV, Jeon M, et al. Gold nanocage-photosensitizer conjugates for dual-modal image-guided enhanced photodynamic therapy. Theranostics. 2014;4(2):163-174. 
172. Huang P, Lin J, Wang SJ, et al. Photosensitizer-conjugated silicacoated gold nanoclusters for fluorescence imaging-guided photodynamic therapy. Biomaterials. 2013;34(19):4643-4654.

173. Nair LV, Nazeer SS, Jayasree RS, Ajayaghosh A. Fluorescence imaging assisted photodynamic therapy using photosensitizer-linked gold quantum clusters. ACS Nano. 2015;9(6):5825-5832.

174. Broering R, Real CI, John MJ, et al. Chemical modifications on siRNAs avoid Toll-like-receptor-mediated activation of the hepatic immune system in vivo and in vitro. Int Immunol. 2014;26(1):35-46.

175. Longmire M, Choyke PL, Kobayashi H. Clearance properties of nanosized particles and molecules as imaging agents: considerations and caveats. Nanomedicine. 2008;3(5):703-717.

176. Li W, Szoka FC Jr. Lipid-based nanoparticles for nucleic acid delivery. Pharm Res. 2007;24(3):438-449.

177. Guo JF, Bourre L, Soden DM, O’Sullivan GC, O’Driscoll C. Can non-viral technologies knockdown the barriers to siRNA delivery and achieve the next generation of cancer therapeutics? Biotechnol Adv. 2011;29(4):402-417.

178. Kumar A, Zhang X, Liang XJ. Gold nanoparticles: emerging paradigm for targeted drug delivery system. Biotechnol Adv. 2013;31(5): 593-606.

179. Guo JF, Rahme K, Fitzgerald KA, Holmes JD, O’Driscoll CM. Biomimetic gold nanocomplexes for gene knockdown: will gold deliver dividends for small interfering RNA nanomedicines? Nano Res. 2015; 8(10):3111-3140.

180. Chwalek K, Bray LJ, Werner C. Tissue-engineered 3D tumor angiogenesis models: potential technologies for anti-cancer drug discovery. Adv Drug Deliver Rev. 2014;79-80:30-39.

181. Quail DF, Joyce JA. Microenvironmental regulation of tumor progression and metastasis. Nat Med. 2013;19(11):1423-1437.
182. Beck B, Blanpain C. Unravelling cancer stem cell potential. Nat Rev Cancer. 2013;13(10):727-738.

183. Moghimi SM, Hunter AC, Andresen TL. Factors controlling nanoparticle pharmacokinetics: an integrated analysis and perspective. Annu Rev Pharmacol. 2012;52:481-503.

184. Zhou C, Long M, Qin YP, Sun XK, Zheng J. Luminescent gold nanoparticles with efficient renal clearance. Angew Chem Int Edit. 2011; 50(14):3168-3172.

185. Hirn S, Semmler-Behnke M, Schleh C, et al. Particle size-dependent and surface charge-dependent biodistribution of gold nanoparticles after intravenous administration. Eur J Pharm Biopharm. 2011; 77(3):407-416.

186. Alric C, Miladi I, Kryza D, et al. The biodistribution of gold nanoparticles designed for renal clearance. Nanoscale. 2013;5(13): 5930-5939.

187. Simpson CA, Salleng KJ, Cliffel DE, Feldheim DL. In vivo toxicity, biodistribution, and clearance of glutathione-coated gold nanoparticles. Nanomedicine. 2013;9(2):257-263.

188. Zhang XD, Wu D, Shen X, Liu PX, Fan FY, Fan SJ. In vivo renal clearance, biodistribution, toxicity of gold nanoclusters. Biomaterials. 2012;33(18):4628-4638.

189. Khlebtsov N, Dykman L. Biodistribution and toxicity of engineered gold nanoparticles: a review of in vitro and in vivo studies. Chem Soc Rev. 2011;40(3):1647-1671.

190. Xie J, Lee S, Chen XY. Nanoparticle-based theranostic agents. Adv Drug Deliver Rev. 2010;62(11):1064-1079.

191. Hanahan D, Weinberg RA. Hallmarks of cancer: the next generation. Cell. 2011;144(5):646-674.

192. Xia Y, Li W, Cobley CM, et al. Gold nanocages: from synthesis to theranostic applications. Acc Chem Res. 2011;44(10):914-924.
International Journal of Nanomedicine

\section{Publish your work in this journal}

The International Journal of Nanomedicine is an international, peerreviewed journal focusing on the application of nanotechnology in diagnostics, therapeutics, and drug delivery systems throughout the biomedical field. This journal is indexed on PubMed Central, MedLine, CAS, SciSearch ${ }^{\circledR}$, Current Contents ${ }^{\circledR} /$ Clinical Medicine,

\section{Dovepress}

Journal Citation Reports/Science Edition, EMBase, Scopus and the Elsevier Bibliographic databases. The manuscript management system is completely online and includes a very quick and fair peer-review system, which is all easy to use. Visit http://www.dovepress.com/ testimonials.php to read real quotes from published authors. 